ARTICLE

\title{
Single cell multi-omic analysis identifies a Tbx1- dependent multilineage primed population in murine cardiopharyngeal mesoderm
}

Hiroko Nomaru1, Yang Liu1,10, Christopher De Bono ${ }^{1,10}$, Dario Righelli ${ }^{2,3}$, Andrea Cirino ${ }^{4,5}$, Wei Wang 6 , Hansoo Song (1) 1, Silvia E. Racedo ${ }^{1}$, Anelisa G. Dantass ${ }^{1,7}$, Lu Zhang ${ }^{8}$, Chen-Leng Cai ${ }^{8}$, Claudia Angelini $^{2}$, Lionel Christiaen (10 ${ }^{6}$, Robert G. Kelly ${ }^{9}$, Antonio Baldini ${ }^{4,5}$, Deyou Zheng $\mathbb{D}^{1}$ \& Bernice E. Morrow (D) ${ }^{1 凶}$

The poles of the heart and branchiomeric muscles of the face and neck are formed from the cardiopharyngeal mesoderm within the pharyngeal apparatus. They are disrupted in patients with 22q11.2 deletion syndrome, due to haploinsufficiency of TBX1, encoding a T-box transcription factor. Here, using single cell RNA-sequencing, we now identify a multilineage primed population within the cardiopharyngeal mesoderm, marked by Tbx 1 , which has bipotent properties to form cardiac and branchiomeric muscle cells. The multilineage primed cells are localized within the nascent mesoderm of the caudal lateral pharyngeal apparatus and provide a continuous source of cardiopharyngeal mesoderm progenitors. Tbx 1 regulates the maturation of multilineage primed progenitor cells to cardiopharyngeal mesoderm derivatives while restricting ectopic non-mesodermal gene expression. We further show that TBX1 confers this balance of gene expression by direct and indirect regulation of enriched genes in multilineage primed progenitors and downstream pathways, partly through altering chromatin accessibility, the perturbation of which can lead to congenital defects in individuals with 22q11.2 deletion syndrome.

\footnotetext{
${ }^{1}$ Department of Genetics, Albert Einstein College of Medicine, Bronx, NY, USA. ${ }^{2}$ Institute for Applied Computing, National Research Council, Naples, Italy

${ }^{3}$ Department of Statistical Sciences, University of Padova, Padova, Italy. ${ }^{4}$ Department of Molecular Medicine and Medical Biotechnology, University Federico II School of Medicine, Naples, Italy. ${ }^{5}$ Institute of Genetics and Biophysics, National Research Council, Naples, Italy. ${ }^{6}$ Center for Developmental Genetics, Department of Biology, New York University, New York, NY, USA. ${ }^{7}$ Federal University of Sao Paulo, Sao Paulo, Brazil. ${ }^{8}$ Department of Pediatrics, Indiana University School of Medicine, Indianapolis, IN, USA. ${ }^{9}$ Aix-Marseille University, CNRS UMR 7288, IBDM, Marseille, France. ${ }^{10}$ These authors contributed equally: Yang Liu, Christopher De Bono. ${ }^{凶}$ email: bernice.morrow@einsteinmed.org
} 
$\mathrm{T}$ he heart develops from two successive waves of mesodermal progenitor cells during early embryogenesis. The first heart field (FHF) constitutes the first wave of cardiac progenitors and results in the primitive beating heart, while the second heart field (SHF) forms the second wave that builds upon the two poles of the heart ${ }^{1,2}$. The SHF can be anatomically partitioned to the anterior SHF $\left(\mathrm{aSHF}^{3,4}\right)$ and posterior SHF $\left(\mathrm{pSHF}^{4-6}\right)$, whose cells migrate to the heart via the dorsal pericardial wall to the outflow tract or inflow tract, respectively. Expression of Mesp1 at gastrulation marks the earliest mesodermal cells that will form the heart ${ }^{7}$. Using single-cell RNAsequencing (scRNA-seq) of the Mesp1 lineage, it was discovered that the FHF, aSHF, and pSHF are specified at gastrulation ${ }^{8}$.

Retrospective clonal analysis ${ }^{9,10}$ and lineage tracing studies ${ }^{11}$ revealed that the branchiomeric skeletal muscles (BrM) of the craniofacial region and neck share a clonal relationship with the SHF. The bipotent nature of these cardiac and skeletal muscle progenitor cells is supported by studies in the ascidian, Ciona, an invertebrate chordate, in which single cells gives rise to both cardiac and skeletal muscle cells ${ }^{12}$. When taken together, a new term, cardiopharyngeal mesoderm (CPM), was introduced to clearly include both SHF cardiac and skeletal muscle progenitor populations $s^{2}$. A cartoon of these populations in the mouse embryo is shown in Fig. 1a. The Tbxl gene, encoding a T-box transcription factor, and gene haploinsufficient in 22q11.2 deletion syndrome (22q11.2DS), is expressed in the CPM and is required for cardiac outflow tract and BrM development ${ }^{2}$, implicating its essential roles in the CPM.

A total of $60-75 \%$ of patients with 22q11.2DS have cardiac outflow tract defects, which often require life-saving surgery during the neonatal period ${ }^{13}$. In addition, most individuals with this condition have speech, feeding, and swallowing difficulties in infancy, due in part to BrM hypotonia ${ }^{14}$. Further, heterozygous mutations of TBX1 in rare, non-deleted individuals, phenocopy symptoms of the deletion ${ }^{15}$. Inactivation of both alleles of $T b \times 1$ in the mouse results in a persistent truncus arteriosus ${ }^{16-18}$ and significant failure to form $\mathrm{BrMs}^{19}$. Gene expression profiling of wild-type versus $T b x 1$ global null mutant embryos identified genes that changed in expression but it was unclear whether the changes were autonomous in the CPM or in other cell populations, such as neural crest cells ${ }^{20,21}$. We therefore do not yet understand the functions of $T b x 1$ on a single-cell level, which is needed to elucidate the true molecular pathogenesis of 22q11.2DS.

The CPM is distributed throughout the embryonic pharyngeal apparatus during early gestation. The pharyngeal apparatus consists of individual bulges of cells termed arches that form in a rostral to caudal manner from mouse embryonic days (E)8-10.5. The formation of individual arches is a highly dynamic but regulated process that requires sufficient cell populations to elongate the OFT and to form diverse BrMs. The cellular and molecular mechanisms of how CPM cells in the pharyngeal apparatus both are maintained in a progenitor state and are allocated to form the heart and BrMs in mammals are unknown.

To fill these gaps, we performed scRNA-seq of mesodermal cell lineages from the pharyngeal apparatus plus heart at multiple stages during embryogenesis. We discovered a multilineage primed progenitor (MLP) population within the CPM, which is maintained and gradually matures from E8-10.5 and has differentiation branches toward cardiac and skeletal muscle fates, serving as common lineage progenitors. MLP cells are localized to the nascent lateral mesoderm of the pharyngeal apparatus, deploying cells to the heart and BrMs. We found that the Tbxi cell lineage marks MLPs and TBX1 activity is critical for their function. Inactivation of Tbxl disrupts a MLP gene expression program needed for differentiation and results in ectopic expression of non-mesoderm genes. We further identify the gene regulatory network downstream of Tbx1 in the MLPs providing insights into the molecular mechanism of mammalian CPM function, essential for understanding the etiology of 22q11.2DS.

\section{Results}

Identification of common progenitor cells in the CPM. To identify the various populations that constitute the CPM (Fig. 1a), we performed droplet-based scRNA-seq on fluorescenceactivated cell sorted GFP expressing cells from microdissected Mesp1 ${ }^{\text {Cre }}$;ROSA26-GFPf/+ (f, flox) embryos at E8.0, E8.25, E9.5, and E10.5 (Table 1, Fig. 1b, Supplementary Figs. 1a, b, 2a, b, 3, and Supplementary Table 1). These stages were chosen because they are the critical periods when $T b x 1$ is expressed, reaching the highest expression at E9.5, and when the pharyngeal apparatus is dynamically elongating; this is coordinated with heart development and BrM specification. To better understand the developmental sequence of events, we integrated the four time point datasets (Fig. 1c) and identified 20 cell clusters (Fig. 1d). Utilizing knowledge of the expression of known marker genes in each cluster (Fig. 1e, Supplementary Fig. 2c-e, and Supplementary Data 1), we identified cell types of all the clusters, half of which include cardiovascular progenitor cell populations or their derivatives (Fig. 1d, bold font).

We identified CPM clusters (C1, C3, C9, C15, and C18) with marker gene expression for the CPM including Tbx1, Isl1, Wnt5a, and Tcf21, among other genes ${ }^{1,22}$ (Fig. 1f, $g$ and Supplementary Fig. 2e). Cluster C9 contains BrM progenitor cells identified by expression of $T c f 21, L h x 2$, and $M y f 5^{22}$ (Fig. 1f and Supplementary Fig. 2e). Clusters $\mathrm{C} 1$ and $\mathrm{C} 18$ contain pSHF populations as identified by expression of Hoxb $1^{5}, T b x 5$, Foxf 1 , and $W n t 2^{23,24}$ (Fig. If and Supplementary Fig. 2e). Many of the pSHF cells are located more medially and caudally in the embryo and contribute to posterior organ development, such as the formation of the lung ${ }^{23,24}$. The cells in C3 express Nkx2-5 and Mef2 $c^{25}$ and cardiac structural protein genes (Tnnt2, Tnni1, Myl4 (Fig. If and Supplementary Fig. 2e)). In addition, subdomains express either FHF genes (Tbx5 but not Isl1 or Tcf21) or aSHF genes (Fgf10, Isl1, Tcf21 but not Tbx5 (Fig. 1h)). We discovered that cluster C15 expresses genes shared by CPM clusters, including Tbxl, Isl1, Wnt5a, Mef2c, Tcf21, and Foxf1 (Fig. If and Supplementary Fig. 2e). Based upon this, we suggest that C15 as a multilineage primed progenitor (MLP) population within the CPM.

Multilineage primed progenitors of the CPM differentiate into cardiac and skeletal muscles. We next investigated the relationship between the MLPs and more differentiated CPM cells using partition-based graph abstraction (PAGA) ${ }^{26}$. Several clusters that are not part of the CPM and were already well separated in the above cluster analysis (C4, C5, C7, C13, and C17), were excluded from PAGA analysis (Supplementary Fig. 2d). The PAGA analysis partitioned the CPM cells into five branches (Fig. 2a and Supplementary Fig. 4a), connecting all mesodermal cell populations. The five branches include cardiomyocytes (CMs), pSHF with lung progenitor cells (pSHF, Lung PC), connective tissue (CT), branchiomeric muscle progenitor cells (BrM), and skeleton/limb (Sk/L). Convergent results from pseudotime analysis (Fig. 2b) and real-time point information (Fig. $2 \mathrm{c}$ and Supplementary Fig. 4b), infer that MLP cells (C15) in the center are in a progenitor state of the CPM while more differentiated cells are toward the outside in each branch (BrM, C9; CMs with aSHF, C3; part of the pSHF, C1 + C18; Fig. 2d and Supplementary Fig. 4c). The MLP cells exist not only in early time points (E8.0, E8.25) but also in later time points (E9.5, E10.5), but with fewer cells at E10.5 (Fig. 2e). 
a

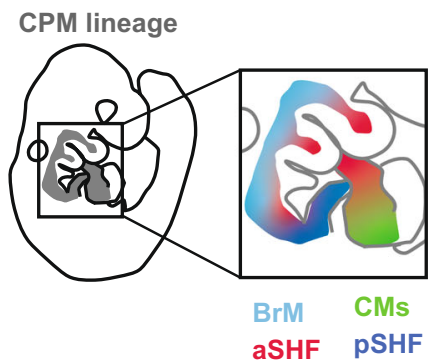

b

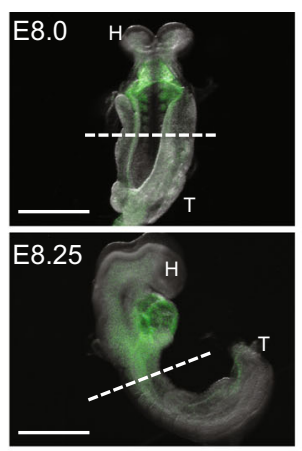

C

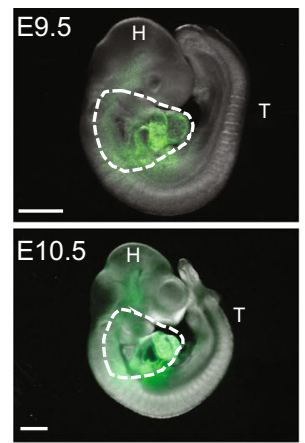

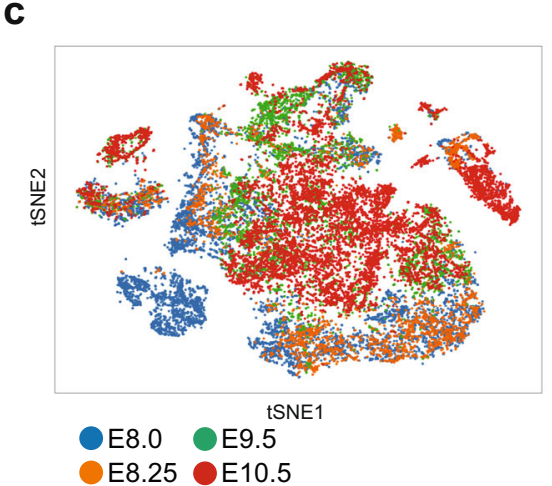

d

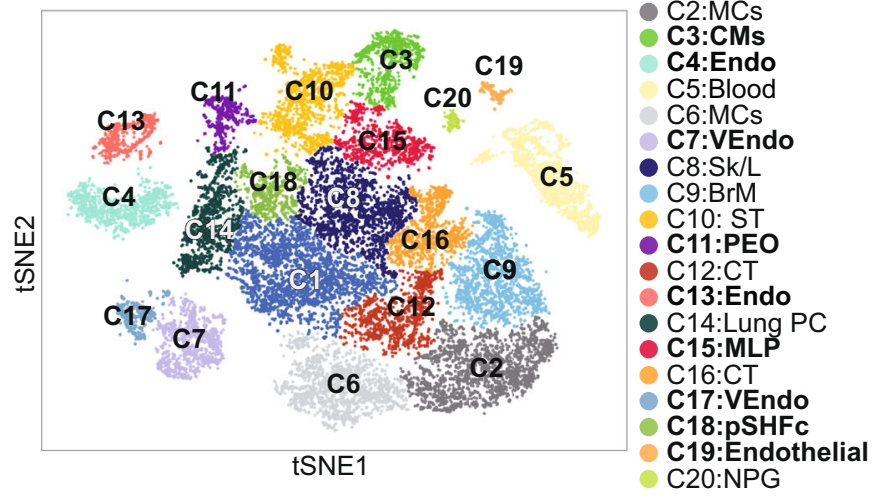

e

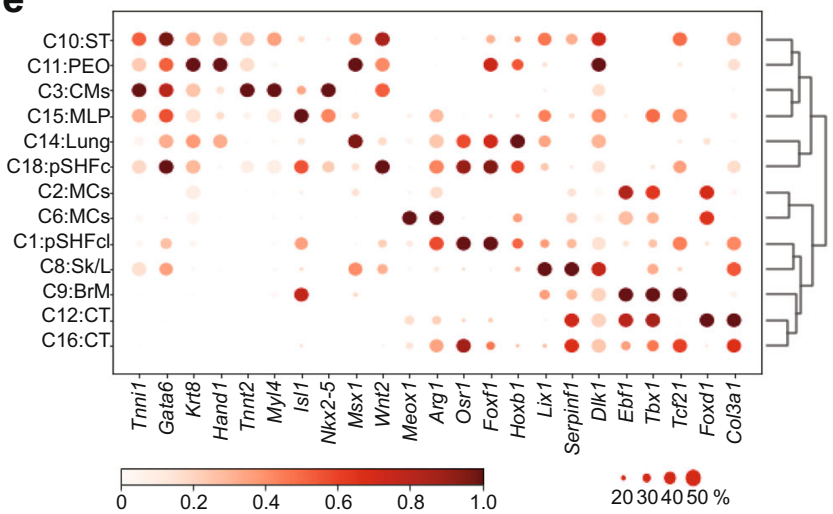

$\mathbf{f}$

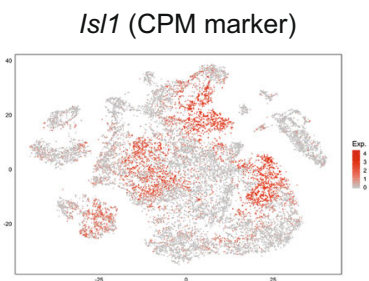

Ebf1 (BrM marker)

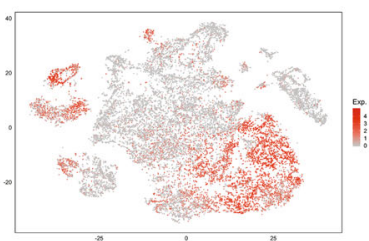

Mef2c (aSHF marker)

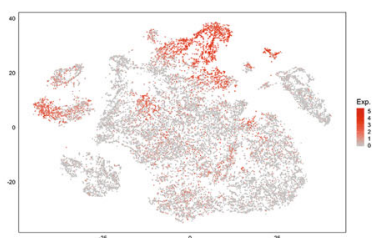

Tbx5 (pSHF/CMs marker)

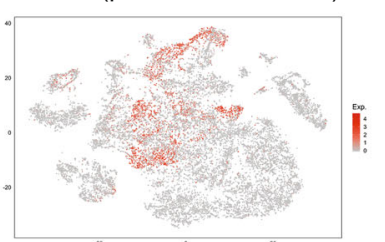

Tcf21 (CPM marker)

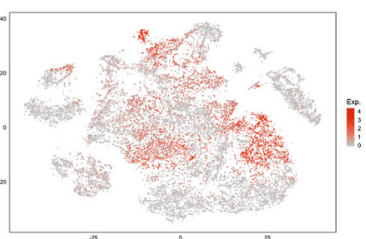

Lhx2 (BrM marker)

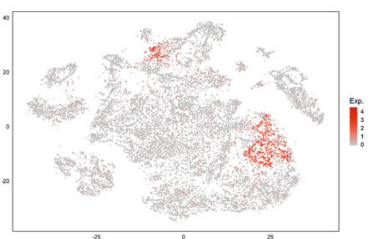

Nkx2-5 (CMs marker)

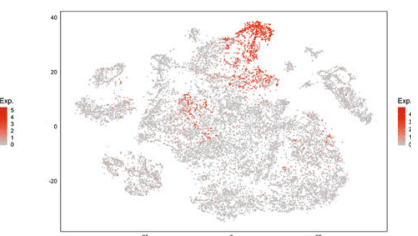

Foxf1 (pSHF marker) g

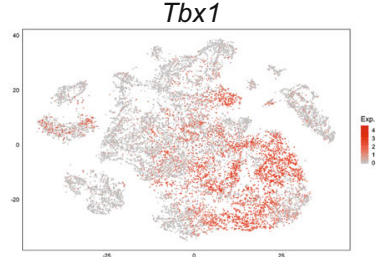

h

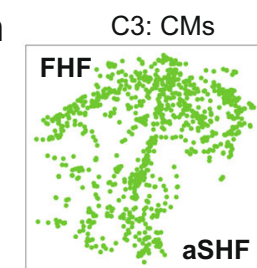

$\mid s / 1$

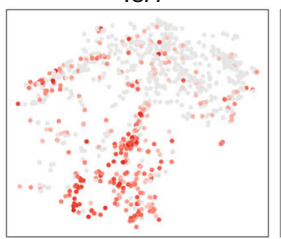

Tbx5

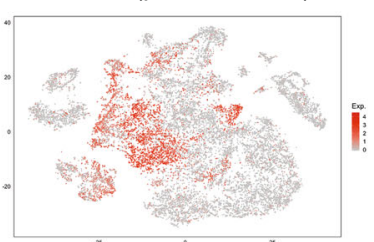

Based on the specific expression of genes (Supplementary Data 1) and distribution of gene expression patterns in the PAGA plot (Fig. 2f and Supplementary Fig. 4d-f), we focused on two marker genes for MLPs, Aplnr (Apelin receptor) and Nrg1 (Neuregulin 1). Aplnr is expressed in the $\mathrm{CPM}^{27}$ but not known for the specific presence in MLPs, while Nrg1 is not known to be a CPM gene, but it is required in the embryonic heart for the development of the chamber myocardium ${ }^{28}$. We examined the co-expression of genes in the cells in CPM populations. The heatmap in Fig. 2g (Supplementary Fig. 4e, f) shows the expression of genes enriched in MLPs, with the same genes also expressed in more differentiated CPM populations (BrM, CMs, and $\mathrm{pSHF}$ ), but at different expression levels, indicating that they are multilineage primed. Taken together, these results further support the progenitor status of MLPs. 
Fig. 1 Single-cell analysis of Mesp1 + lineages at E8.0 to E10.5 identifies CPM lineages. a Cartoon of an E9.5 embryo in a right lateral view (left, CPM, gray). The CPM includes the branchiomeric muscle progenitor cells (BrM; aqua), anterior SHF (aSHF; red), and part of the posterior SHF (pSHF; blue). Because cardiomyocyte progenitors (CMs; green) are from both the FHF and CPM, only CPM-derived CMs are included. b Whole-embryo images with GFP fluorescence of Mesp1Cre, ROSA26-GFPf/+ embryos at E8.0, E8.25, E9.5, and E10.5 used for scRNA-seq. The white dotted line represents the region that was dissected. Only GFP-positive cells were used for scRNA-seq. H head, T tail. Scale bar E8.0, E8.25 is $750 \mu \mathrm{m}$; E9.5 and E10.5 is $500 \mu \mathrm{m}$. c t-distributed stochastic neighbor embedding (tSNE) plot colored by developmental stage. $\mathbf{d}$ tSNE plot colored by clusters (C1-20). Cardiac relevant clusters are in bold font. C1: pSHFcl, posterior CPM includes cardiac and lung progenitor cells; C2: MCs, mesenchyme expressing epithelial-mesenchymal transition markers; C3: CMs cardiomyocyte progenitor cells; C4: Endo endocardium and endothelial cells; C5: Blood blood cells; C6: MCs mesenchyme expressing epithelial-mesenchymal transition markers; C7: VEndo vascular endothelial cells; C8: Sk/L skeleton/limb progenitor cells; C9: BrM branchiomeric muscle progenitor cells; C10: ST septum transversum progenitor cells; C11: PEO proepicardial organ; C12: CT connective tissue progenitor cells; C13: Endo endothelial cells; C14: Lung PC lung progenitor cells; C15: MLP multilineage progenitor cells; C16: CT connective tissue progenitor cells; C17: VEndo vascular endothelial cells; C18: pSHFc posterior CPM of cardiac progenitor cells; C19: Endothelial cells; C20: NPG neural progenitor cells. e Heatmap of average gene expression of marker genes in representative clusters. Dot size indicates the fraction of cells expressing the genes in each cluster and color indicates scaled mean expression (bar below; dark red is the strongest expression). $\mathbf{f}, \mathbf{g}$ tSNE plots showing expression of CPM marker genes (f) and Tbx 1 (g). The color spectrum from gray to red indicates expression levels from low to high. $\mathbf{h}$ tSNE plots of CM progenitors showing the expression level of CM (Tbx5) and CPM (Is/1) marker genes.

Table 1 Summary of scRNA-seq samples.

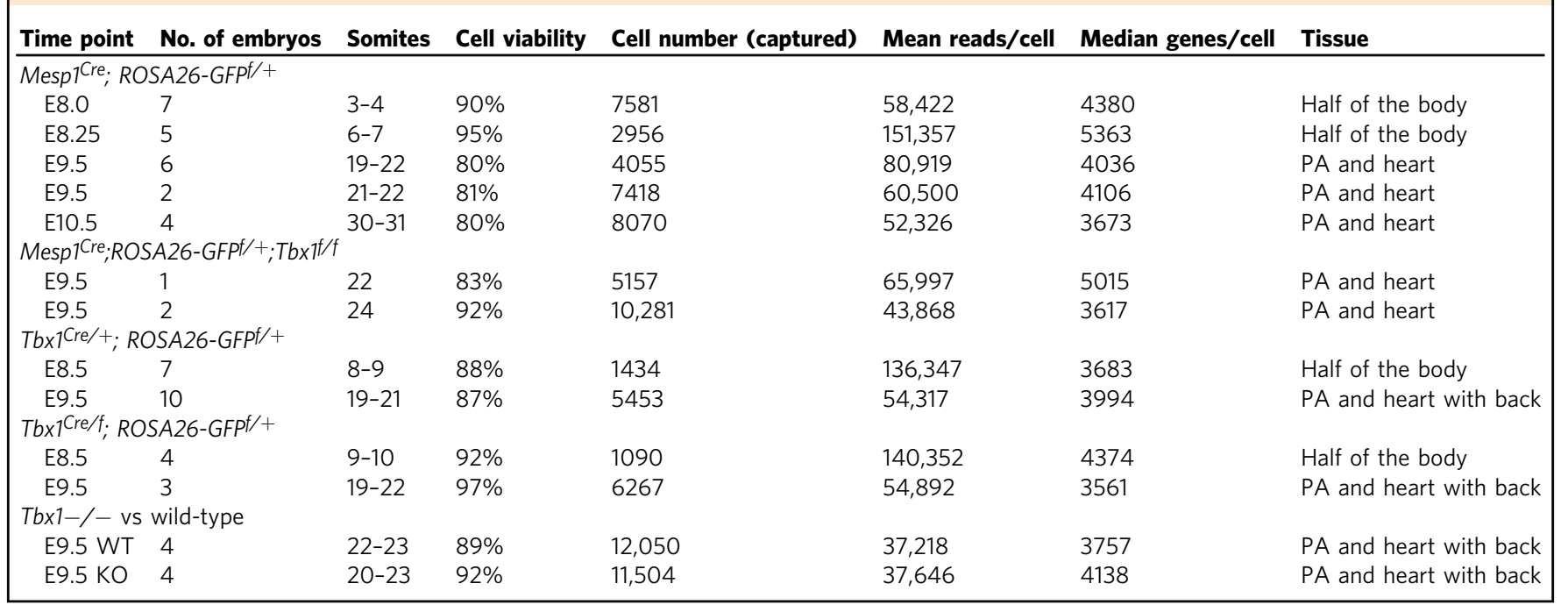

The MLPs are bilaterally localized to the caudal pharyngeal apparatus. To elucidate whether MLPs are localized within a defined embryonic region in the pharyngeal apparatus, we performed RNAscope in situ hybridization analysis using probes for genes enriched in MLPs including Tbx1, Isl1, Aplnr, and Nrg1 with the Mesp $1+$ lineage marked by EGFP expression (Fig. $2 \mathrm{~h}-\mathrm{m}$ ). The pharyngeal arches form in a rostral to the caudal manner where the most caudal and lateral mesoderm is the least differentiated, while the rostral mesoderm has already migrated to the core of the arch to form BrM progenitor cells or toward the dorsal pericardial wall and poles of the heart ${ }^{29,30}$. In embryos at E8.5 and E9.5, Nrg1 and Aplnr co-expressing cells are found bilaterally in the lateral part of the caudal pharyngeal apparatus, containing nascent mesoderm that is not yet differentiated to cardiac or skeletal muscle (Fig. $2 \mathrm{~h}-\mathrm{j}$ and Supplementary Fig. 5a, b). At E8.5, Nrg1 and Aplnr are expressed in these regions within the forming second arch (Fig. 2i) and at E9.5, by the forming fourth arch (Fig. 2j), both overlapping with Isll expression. The Mesp $1+$ lineage is marked with EGFP expression in the second arch at E8.5 and the fourth arch at E9.5 (Fig. 2k and Supplementary Fig. 5c, d). Tbx1 and Isl 1 are also expressed in those regions (Fig. 2l, m). We suggest that the MLPs remain in the same region of the caudal pharyngeal apparatus, while they deploy cells rostrally, medially, and dorsally thereby explaining in part the mechanism for the extension of the pharyngeal apparatus caudally (Fig. 2n).

MLPs dynamically transition over time. An important question is whether MLPs as CPM progenitors, maintain the same state of gene expression over time. To address this, we examined differentially expressed genes in MLPs from E8-10.5. We identified core CPM genes that are expressed similarly at all time points, including Isl1, Mef2c, and Nkx2-5 (Fig. 3a). However, we also found that early expressing genes such as Aplnr, Nrg1, Irx1-5, $F g f 8 / 10$, and $T b x 1$ (Fig. 3b) are reduced over time, with increasing expression of cardiac developmental genes such as Hand2, Gata3/ 5/6, Bmp4, and Sema3c (Fig. 3c). These gradient differences are also visualized in violin plots (Fig. $3 \mathrm{~d}-\mathrm{f}$ ). $N k \times 2-5$ and Sema $3 c$ are expressed in the caudal pharyngeal apparatus at E9.5, like that of Isl1, Tbx1, and Aplnr, defining MLPs (Fig. 3g). The MLP region is reduced in size in the caudal pharyngeal apparatus, pharyngeal arches 3-6, at E10.5 (Fig. 3g). Further, expression of the earlyMLP marker Aplnr is not observed at E10.5, while co-expression of Sema3c, Nkx2-5, and Isl1 occur strongly in the OFT (Fig. 3g). This is consistent with the model that the MLPs continuously allocate progenitor cells to BrMs and OFT-CMs, while showing a gradual maturation themselves by E10.5 (Fig. 3h). 
a

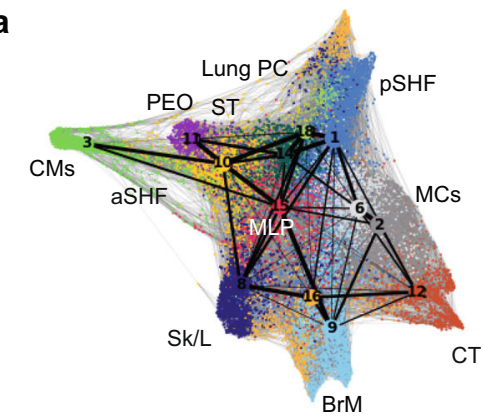

b

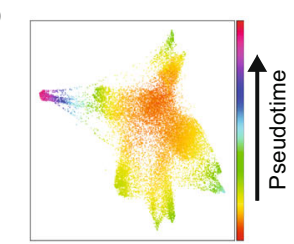

d

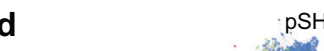

C

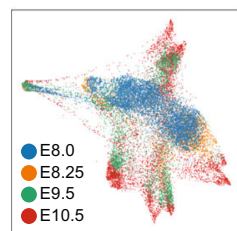

e

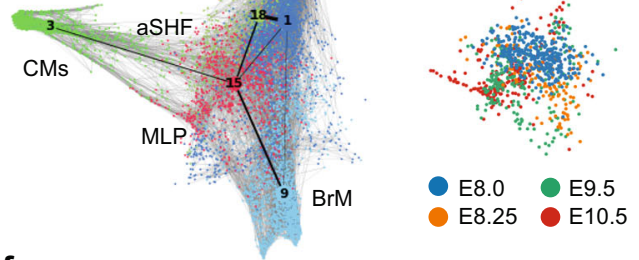

f

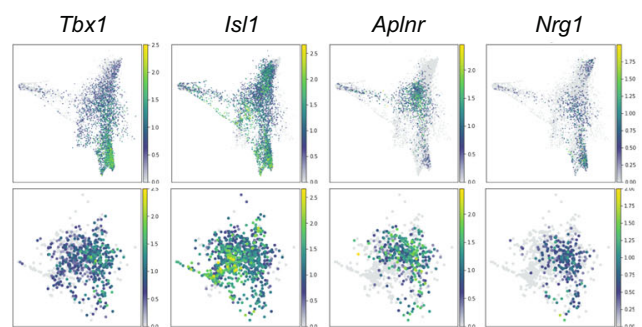

g

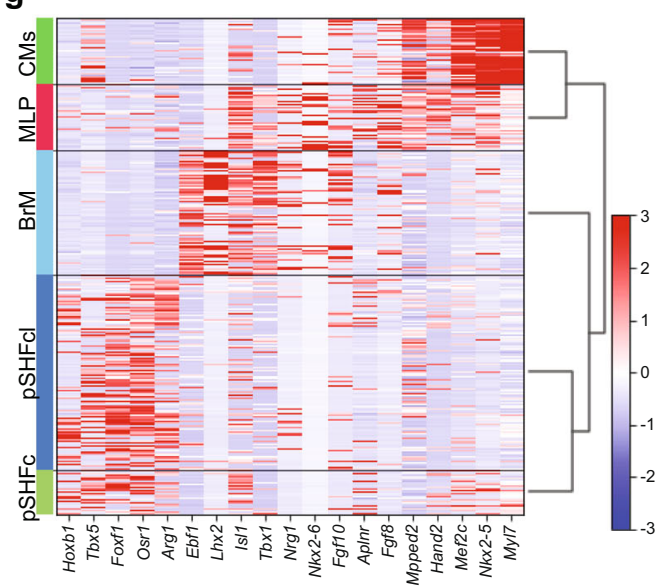

h
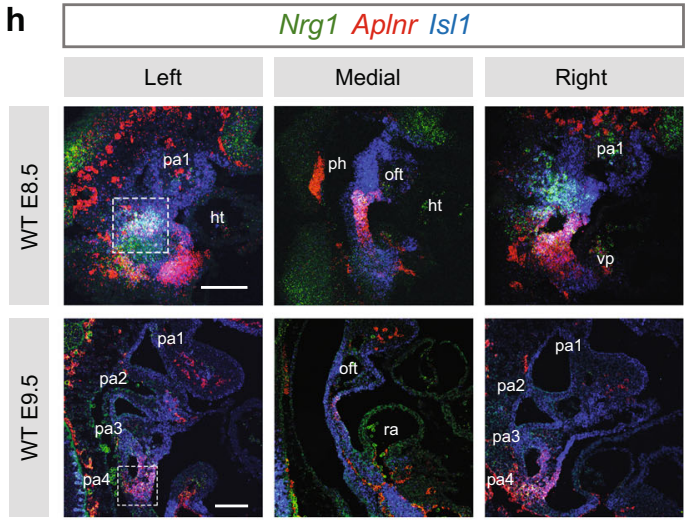

i
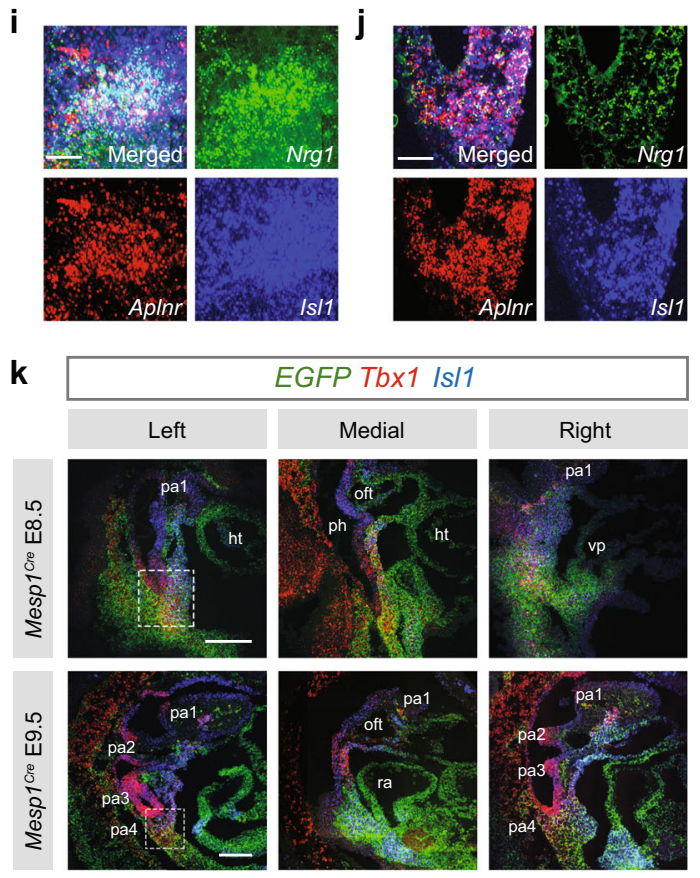

I
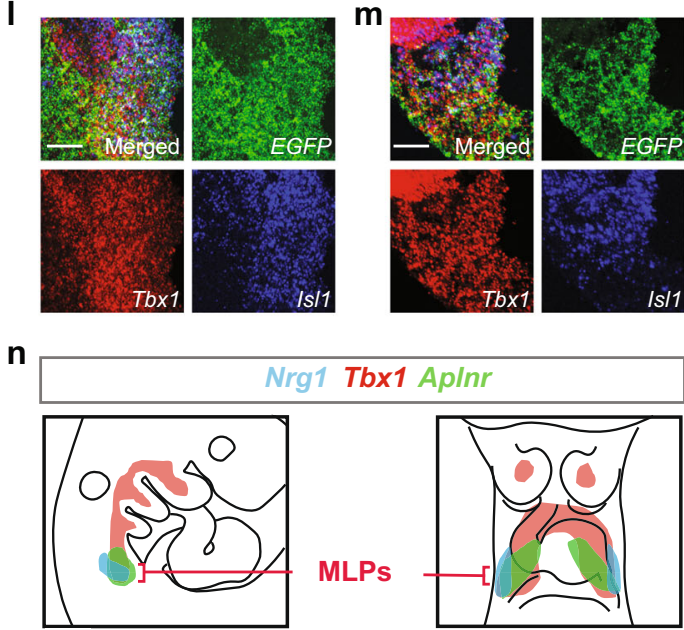

MLPs

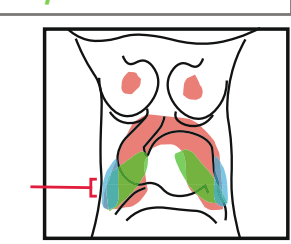

The intersection of the Tbx1 and Mesp1 lineages helps to identify the CPM. Tbx1 function in MLPs and roles in derivative $\mathrm{CPM}$ cells on a single-cell level are unknown. We therefore examined the Mesp1 and Tbx1 lineages in control embryos to understand how the CPM lineages compare in relation to Tbx1. The Mesp1 lineage contributes more broadly to the embryonic mesoderm, while $T b x 1$ is expressed in pharyngeal endoderm and distal pharyngeal ectoderm ${ }^{31}$, in addition to the $\mathrm{CPM}^{32}$ Although Tbx1 is strongly expressed in the CPM, it is not expressed in the heart, neither in the FHF nor the caudal and medial pSHF at the timepoints analyzed ${ }^{33-35}$. The intersection of these two datasets defines the CPM more precisely.

The $T b x 1^{C r e}$ allele has a knockin of Cre that inactivates one copy of the Tbx1 gene. The Tbx $1^{\text {Cre }}$ mice do not have cardiac or 
Fig. 2 MLPs are localized to the caudal pharyngeal apparatus. a Single-cell embedding graph with PAGA plot colored by clusters with abbreviated names identical to those in Fig. 1d. b, c Single-cell embedding graph colored by pseudotime with colors indicating advancing time (b) or real time of samples (c). d Single-cell embedding graph with PAGA plot of the CPM clusters. e Single-cell embedding graph of only MLPs colored by stage. f Single-cell embedding graph of the CPM (above) and MLPs (below) colored by gene expression level (gray to blue, green, yellow, low to high). $\mathbf{g}$ Heatmap of expression of genes enriched in the CPM. Row indicates the expression per cell (blue to white, to red; low to high). h-j RNAscope in situ hybridization with Nrg1 (green), Aplnr (red), and Is/7 (blue) mRNA probes on sagittal sections from wild-type embryos at E8.5 (upper panel; $n=3$ ) and E9.5 (lower panel; $n=3$ ). Scale bar, $100 \mu \mathrm{m}$. The white dotted line indicates the position in images with higher magnification (i, j; scale bar, $30 \mu \mathrm{m}$ ). $\mathbf{k}$-m RNAscope in situ hybridization with EGFP (green) mRNA probe, marking Mesp1+ lineage cells, Tbx1 (red) and Is/1 (blue) mRNA probes on sagittal sections from Mesp1Cre; ROSA26-GFPf/+ embryos at E8.5 (upper panel; $n=3$ ) and E9.5 (lower panel; $n=3$ ). Scale bar, $100 \mu \mathrm{m}$. The white dotted line indicates the position in images with higher magnification (I, $\mathbf{m}$; scale bar, $30 \mu \mathrm{m}$.). $\mathbf{n}$ Summary of the expression pattern of Nrg1, Tbx1, and Aplnr with respect to the MLPs in the cartoon of a sagittal section. ht heart, oft outflow tract, pa pharyngeal arch, ph pharynx, ra right atrium, vp venous pole. 1, 2, and 3 indicate the first, second, and third pharyngeal arches, respectively.

aortic arch defects in the SwissWebster background ${ }^{36}$. We then performed scRNA-seq of the Tbx1 ${ }^{\text {Cre }}$ lineage at E9.5 and integrated this with data from the Mesp $1^{C r e}$ lineage at the same stage to compare the characteristics of the two lineages (Fig. $4 \mathrm{a}-\mathrm{c}$ and Supplementary Data 2). Data integration provides consistency in defining common cell types among different samples, in addition to removing batch effects ${ }^{37}$. We found that the CPM can be identified in both populations (Fig. $4 \mathrm{a}$, bold font). As expected, the Mesp 1 lineage includes the FHF, which is not included in the Tbx1 lineage, while the Tbx1 lineage includes the pharyngeal epithelia and otic vesicle, not included in the Mesp1 lineage (Fig. $4 \mathrm{~b}, \mathrm{c}$ ). The relative proportions of CPM populations are shown in Fig. $4 \mathrm{~d}$. The pSHF in the Mesp $1^{\text {Cre }}$ lineage includes the caudal pSHF with lung progenitors that is not included in the Tb $x 1^{\text {Cre }}$ lineage ${ }^{31}$. The MLPs are found in both lineages, marked by the expression of Isl1, Aplnr, Tbxl, and Nrg1 (Fig. 4e). Therefore, the data from scRNA-seq using Tbx1 ${ }^{\text {Cre }}$ help define the CPM better and serves as a replication for the data on the CPM from scRNA-seq using Mesp $1^{\text {Cre }}$, which is relevant to Tbx1 as shown in Fig. $4 \mathrm{f}$.

Tbx1 regulates MLP development by promoting gene expression needed for differentiation and restricting the expression of non-mesodermal genes. Mesp1 $1^{C r e}$ and $T b \times 1^{C r e}$ mediated Tbx1 conditional null embryos have similar phenotypes, including hypoplasia of the caudal pharyngeal apparatus and a fully penetrant persistent truncus arteriosus ${ }^{16,32}$. This supports the utility of both Cre lines in this study and the importance of the mesoderm domain of Tbx1 expression in mediating its function. We inactivated Tbx1 in the Mesp1 and Tbx1 lineages (Fig. 5a, b) and performed scRNA-seq to ascertain how its loss affects the CPM, using both lineages as a comparison and replication for each other (Table 1). We generated embryos that were Mesp1 $1^{\mathrm{Cre} /+^{+}}$Tbx $1^{+/+} \quad\left(\right.$ Mesp $\left.1^{\mathrm{Cre}} \mathrm{Ctrl}\right)$ vs Mesp1 ${ }^{\mathrm{Cre} /+} ; \mathrm{Tb} \times 1^{\mathrm{flf}}$ (Mesp1 $\left.{ }^{\text {Cre }} \mathrm{cKO}\right)$ at E9.5 (Supplementary Fig. 1a, b, Fig. 5a, and Table 1) and performed two biological replicates. We also gen-

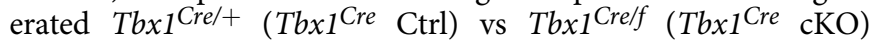
embryos at E8.5 and E9.5 (Supplementary Fig. 1c, d, Fig. 5b, and Table 1). The E8.5 stage used ( $8-10$ somites) is only very slightly different from the E8.25 stage (6-7 somites) used for the

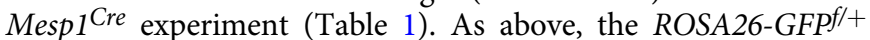
reporter was used to purify the lineages (Supplementary Fig. 1).

With the conditional knockout data, we performed two integrated and clustering analyses; one for Mesp1 $1^{C r e}$ Ctrl vs Mesp1 $1^{C r e} \mathrm{cKO}$ (two replicates; Supplementary Fig. 6 and Supplementary Data 3) and another for Tbx1 Cre Ctrl vs Tbx1Cre cKO embryos (Supplementary Fig. 7 and Supplementary Data 4-5), and, then again focusing on the CPM. With increased resolution afforded by the single time point experiments, we found that the aSHF population is continuous with the somatic mesoderm (SoM; Fig. 5c-f). The SoM is adjacent to the aSHF and in embryos and gives rise to the ventral pericardial wall (expressing Msx1, Msx2, Epha3, but not Nkx2-5;38 Nkx2-5 and $M s \times 2$ are shown in Fig. 5g, h).

The ratio of the number of MLPs compared to the total number of cells in each replicate of $M e s p 1^{\text {Cre }} \mathrm{Ctrl}$ versus $\mathrm{cKO}$ shows a modest variation (first replicate of WT (WT1) is 0.07 (284/4046 cells), WT2 is $0.129(912 / 7048)$ vs first replicate of cKO (KO1) is $0.101(474 / 4689)$ and $\mathrm{KO} 2$ is $0.108(1056 / / 9750)$ ), nonetheless, MLPs are present in both (Fig. $5 \mathrm{c}-\mathrm{f}$ ). To understand how Tbx1 affects gene expression within the MLPs and derivative cell types leading to the observed phenotypes at later stages, we analyzed differentially expressed genes (DEGs) with the scRNAseq datasets. We identified DEGs in each cluster in the two replicates of Mesp1 Ctrl vs cKO embryos (Supplementary Data 4) and separately DEGs in Tbx $1^{\text {Cre }}$ Ctrl vs Tbx $1^{\text {Cre }} \mathrm{cKO}$ embryos at E9.5 (Supplementary Data 6). The DEGs in the two Mesp ${ }^{\text {Cre }} \mathrm{Ctrl}$ vs cKO replicates overlapped significantly, and the final DEGs were from the pooled replicates. Furthermore, to focus on the most reproducible alterations, we examined only DEGs shared in both Mesp1 replicates and between Mesp1 and Tbx1 scRNA-seq datasets at E9.5, which change in the same direction, with adjusted $P$ value $<0.05$ and have an absolute $\log 2$-fold change $>0.25$ (Fig. 6a). In the MLPs, we identified 651 DEGs; 468 genes were decreased and 183 were increased in both Mesp1 and Tbx1 cKO embryos at E9.5 (Fig. 6b, Supplementary Data Gene ontology (GO) analysis was used to identify enriched biological functions of the downregulated genes shared in both Mespl and Tbx1 cKO embryos (Supplementary Data 8). Genes affected in MLPs are involved in cell migration, organ development, and muscle development (Fig. 6c; e.g., Sox9, Mef2c, Grem1, Hey1, Bmp7, Fgf10; Foxf1, Wnt5a). Mef2 $c^{39}$, Hey $1^{40}, B m p 7^{41}$, Fgf $10^{3}$, and $W n t 5 a^{42,43}$ are required for normal cardiac development. Enriched GO terms for the upregulated genes included axonogenesis such as $B d n f^{44}$ or $\operatorname{Pax}^{45}$, inner ear development (placodal formation), and cell fate determination (Fig. 6d). Further, genes not normally expressed in the mesoderm at detectable levels were expressed, such as $\operatorname{Pax}^{46}$ (Fig. 6d). The ratio of the number of Isl1 and Aplnr expressing MLPs in Mesp1 ${ }^{\text {Cre }}$ Ctrl embryos was reduced in Tbx1 cKO embryos (WT1, 0.246 (70/284 cells); WT2, 0.214 (195/912) vs cKO1, 0.011 (5/ 474); cKO2, $0.004(5 / 1,056))$, while the ratio of the number Pax 8 expressing MLPs was dramatically increased (WT1, 0.011 (3/284 cells), WT2, 0.003 (3/912); cKO1, 0.395 (187/474); cKO2, 0.181 $(191 / 1,056))$, further indicating functional changes in MLPs.

To confirm expression changes from the scRNA-seq experiments when Tbx1 is inactivated, we checked the expression pattern of Aplnr and Pax8 in vivo using RNAscope analysis. These genes are at the top fold change among DEGs decreased (Aplnr) or increased (Pax8) in the MLPs of Mesp $1^{\text {Cre }} \mathrm{cKO}$ vs Ctrl embryos, with consistent results in Tbx1 ${ }^{\text {Cre }} \mathrm{Ctrl}$ vs $\mathrm{cKO}$ embryos (Supplementary Data 7). Ectopic Pax8 expression was observed in 
a

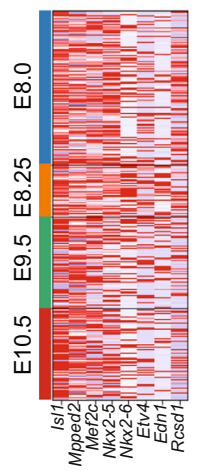

d

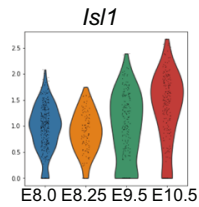

e

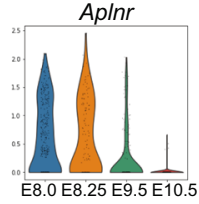

f
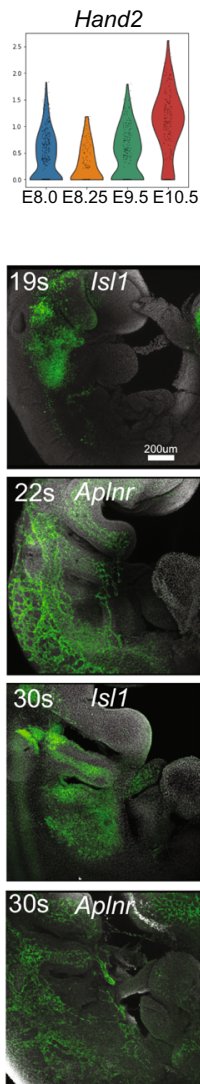

b

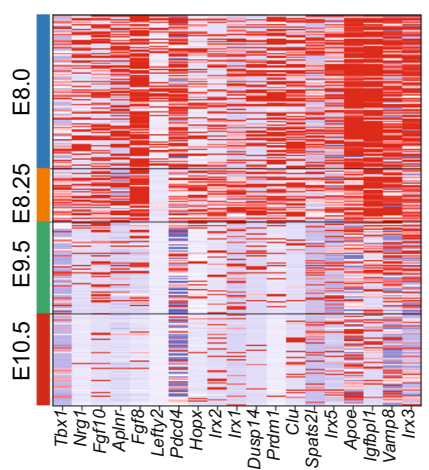

C

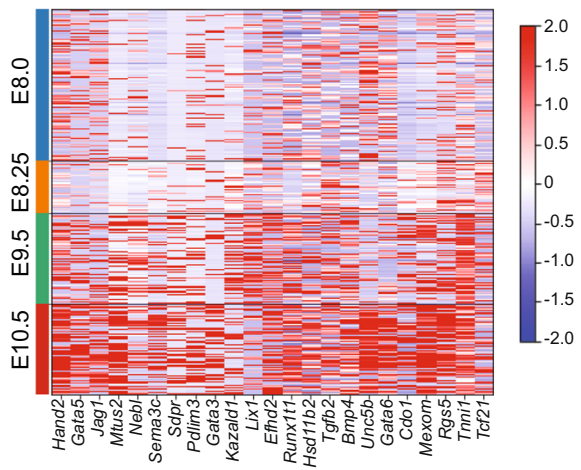

Mpped2

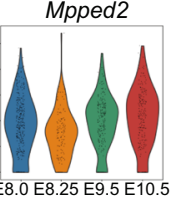

Tbx 1
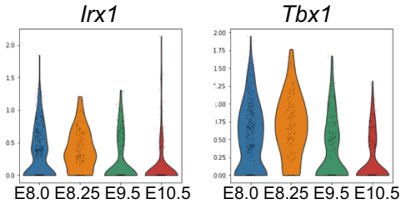

h
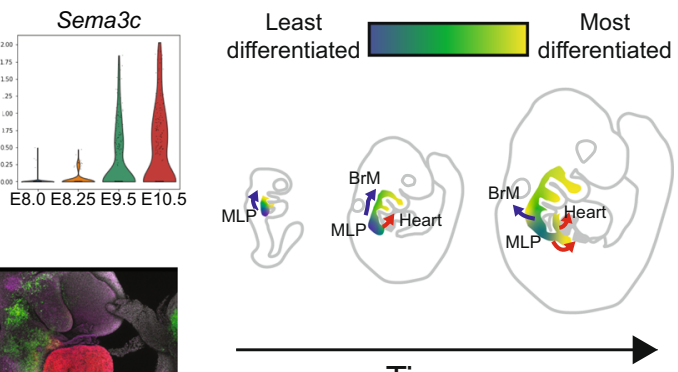

Time

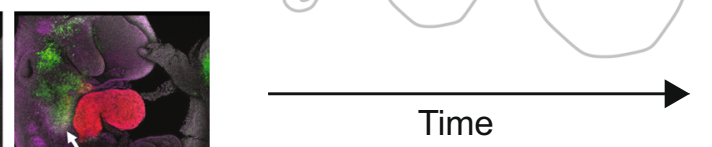

the MLPs of Mesp1 $1^{\text {Cre }}$ and Tbx1 ${ }^{\text {Cre }} \mathrm{cKO}$ embryos (Fig. 6e, f and Supplementary Fig. 8). In serial transverse sections of both sets of cKO and control embryos at E9.5, reduced levels of Aplnr expression in the MLPs in the posterior pharyngeal apparatus were observed, while Pax 8 was increased in the same region containing EGFP + cells (Fig. 6g, h and Supplementary Fig. 9).
We next performed scRNA-seq of wild-type versus $T b \times 1^{-/-}$ embryos at E9.5, without cell sorting, as an independent replication for these experiments, and we found similar gene expression changes as when Mesp $1^{\mathrm{Cre}}$ or $\mathrm{Tb} \times 1^{\mathrm{Cre}}$ lines were used for gene inactivation (Supplementary Fig. 10). We note that in this experiment, the ability to identify individual cell types is reduced 
Fig. 3 MLPs transition as cells are allocated to more differentiated states over time. a Heatmap of expression of core genes in MLPS at E8, E8.25, E9.5, and E10.5. Row indicates the expression of each cell. $\mathbf{b}$ Heatmap of expression of the genes enriched in expression in earlier stage-MLPs (E8, E8.25) and shown in all four stages. Row indicates the expression of each cell. c Heatmap of expression of the genes enriched in expression in intermediate (E9.5) and later stage MLPs (E10.5) and shown in all four stages. Row indicates the expression in each cell. d Violin plots of the expression of core genes (IsI1, Mef2c, Nkx2-5, Mpped2) in MLPs over time. e Violin plots of expression of early-MLP genes (Aplnr, Nrg1, Irx1, Tbx1) in MLPs over time. f Violin plots of expression of late-MLP genes (Hand2, Gata6, Bmp4, Sema3c) in MLPs over time. g Whole embryo RNAscope with Is/1 (green), Nkx2-5 (red), Sema3c (purple), Aplnr (green), and Tbx1 (red) at E9.5 (19 and 22 somites) and E10.5 (30 somites) to identify MLPs in the distal pharyngeal apparatus ( $n=3$ for each stage). Images on the right of each row are a composite of all three probes. V ventricle, OFT cardiac outflow tract, MLP, PA1-4 are indicated. Scale bar, $200 \mu \mathrm{m}$. $\mathbf{h}$ Cartoon of MLP transitions and cell allocation over time. The MLPs in the nascent pharyngeal mesoderm migrate dorsally and will differentiate to BrMs or ventrally and medially to CMs. Blue arrow indicates migration to form BrMs and red arrow(s) indicates migration to the poles of the heart to form CMs. The color spectrum from blue to yellow indicates differentiation from MLPs to their derivative cell types.

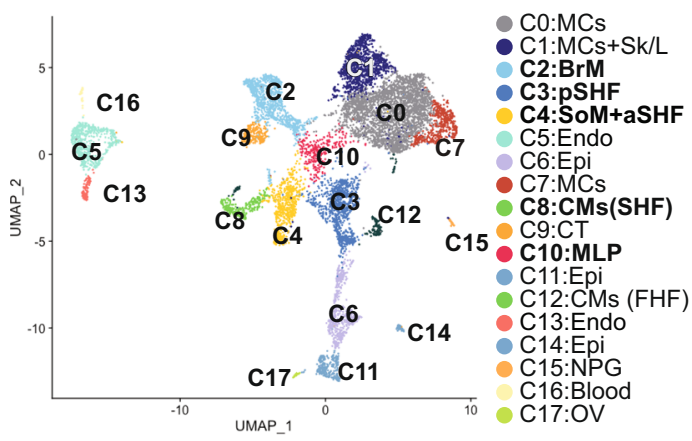

c

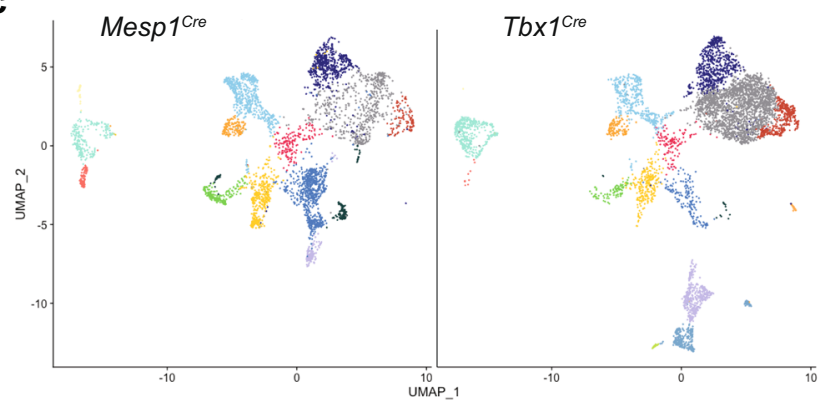

e
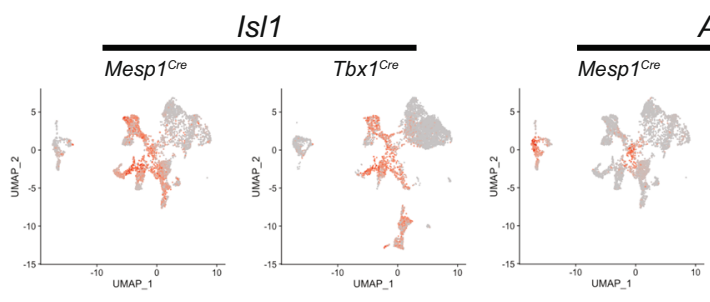

Aplnr

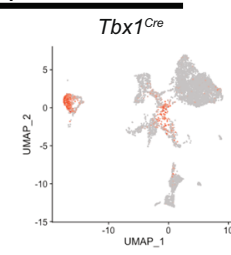

Tbx1
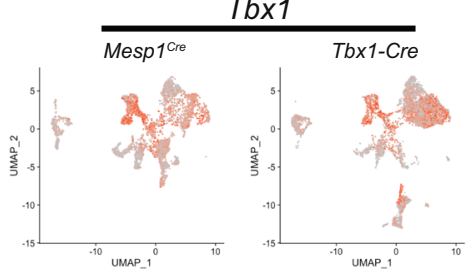

b

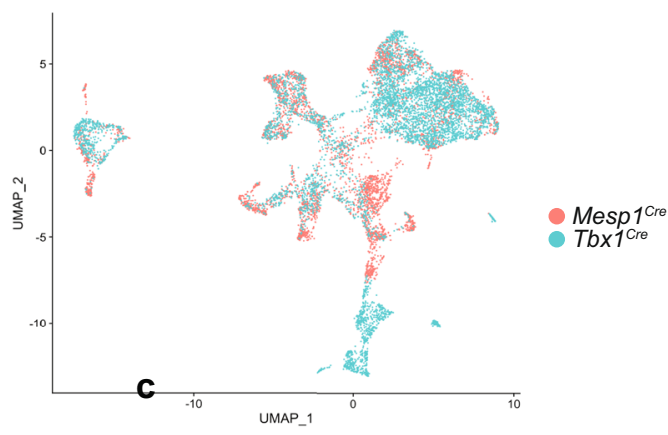

d

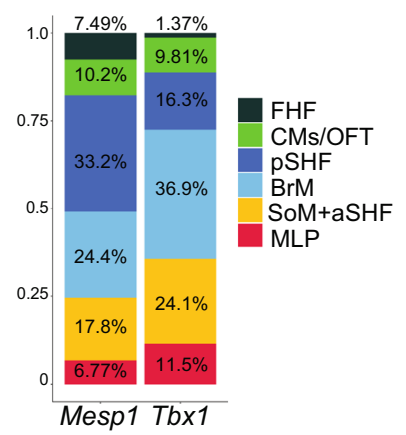

f

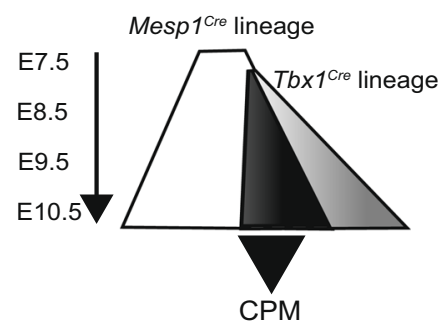

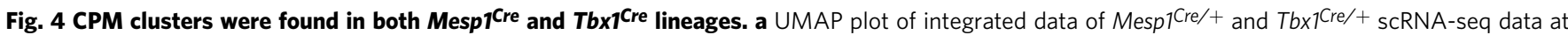
E9.5, colored by cluster. CPM clusters are shown in bold font. b UMAP plot colored by samples (Mesp7Cre, coral; Tbx7 Cre, aqua). c UMAP plots, colored by cluster, separated by samples. $\mathbf{d}$ The ratio of cell populations of CPM lineages in Mesp 7 Cre/ + and Tbx 7 Cre/ + scRNA-seq data. A two proportion $Z$ test was performed in each cluster with 95\% confidence interval (MLP: $P$ value $=2.27 \mathrm{e}-13$; $\mathrm{pSHF}: P$ value $=2.2 \mathrm{e}-16$; SoM/aSHF: $P$ value $=2.72 \mathrm{e}-5$; OFT + CMs: $P$ value $=0.70$ ). e UMAP plots for showing MLP marker genes, separated by samples. The color spectrum from gray to red indicates expression level from low to high. $\mathbf{f}$ Intersection of scRNA-seq data between the two Mesp $7^{C r e}$ and $T b \times{ }_{7}$ Cre populations. Black triangle shows genes shared in both populations and this represents the CPM. Genes shared in both populations have been used for further study in this report. Genes expressed in one versus the other have not been further investigated. 
a Mesp1 $1^{\text {cre }}$
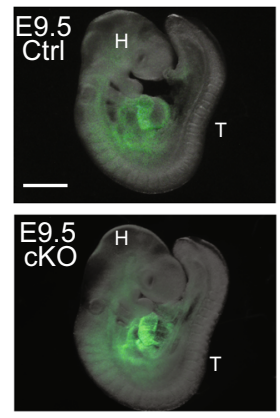

Mesp1 ${ }^{\text {cre }}$

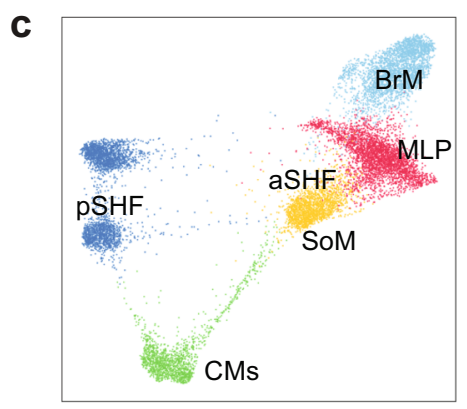

g

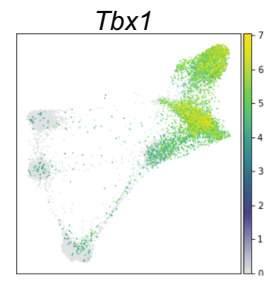

h

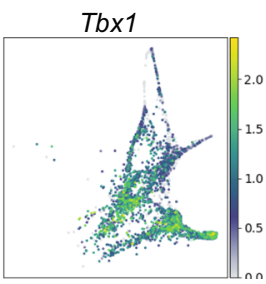

\section{b Tbx $1^{\text {cre }}$}
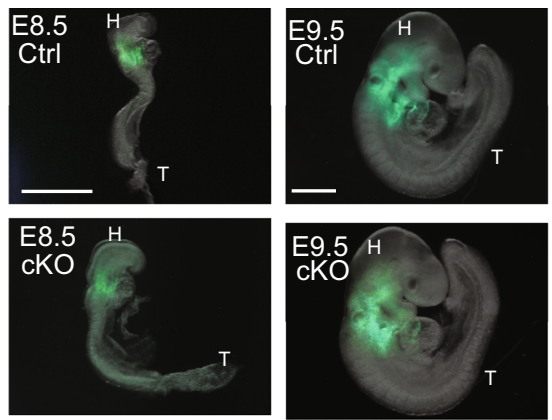

Tbx $1^{\text {cre }}$

d

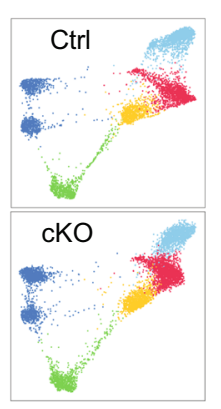

e
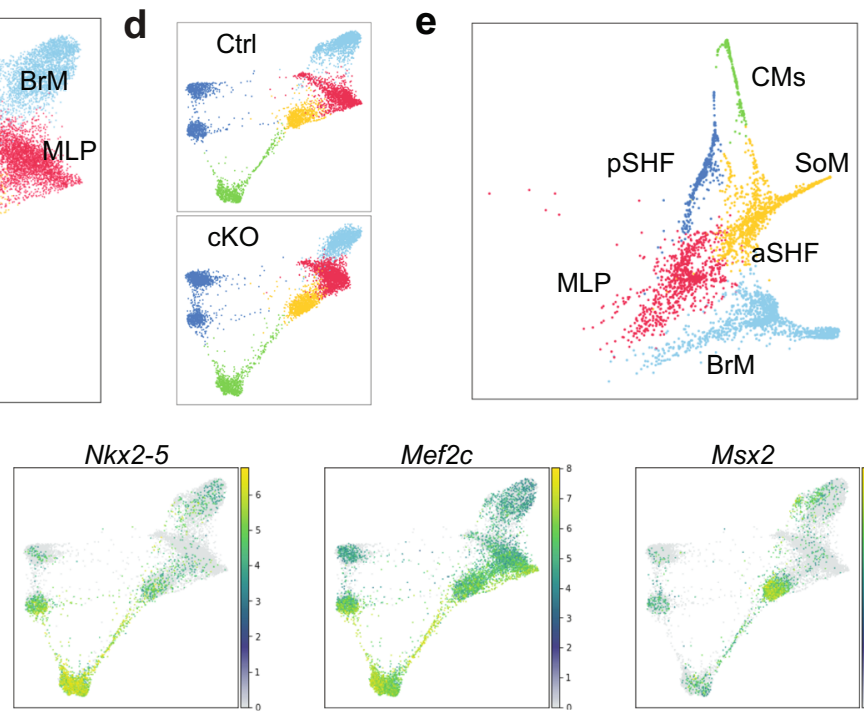

Nkx2-5

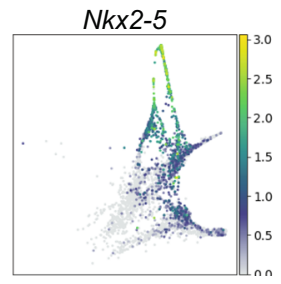

Mef2c

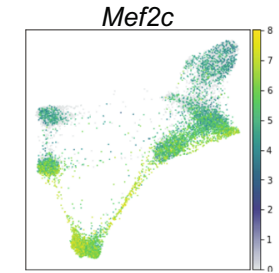

Mef2c

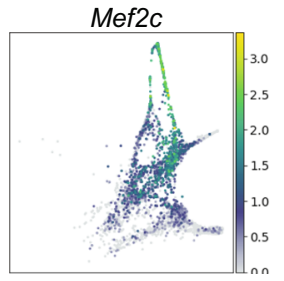

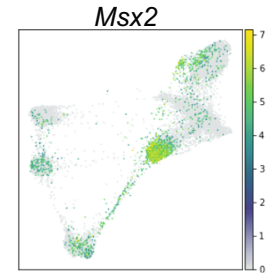

Msx2

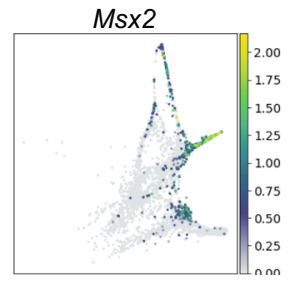

f

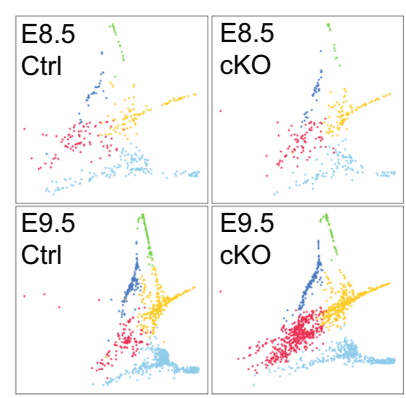

Tbx5

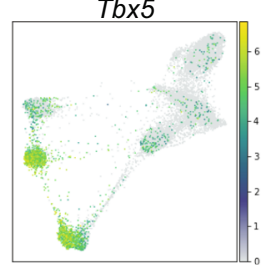

Tbx5

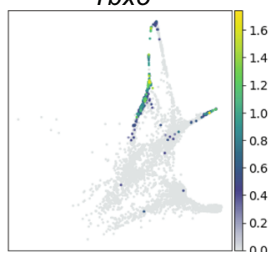

Fig. 5 Tbx1 is required for the progression of MLPs to more differentiated states. a Whole-mount embryo images with GFP fluorescence of Mesp1Cre; ROSA26-GFPf/+ (Ctrl; upper panel, similar to Fig. 1a) and Mesp1 Tre; ROSA26-GFPf/+;Tbx $1^{f / f}$ (cKO; lower panel) embryos at E9.5 used for scRNA-seq. The pharyngeal apparatus and heart were collected. The sorted GFP-positive cells were used for scRNA-seq. $\mathrm{H}$ head, $\mathrm{T}$ tail. Scale bar, $500 \mathrm{\mu m}$. b Whole-embryo images with GFP fluorescence of Tbxicre/+; ROSA26-GFPf/+ (Ctrl; upper panels) and Tbx7 Cre/f; ROSA26-GFPf/+ (cKO; lower panels) embryos at E8.5 (left) and E9.5 (right) used for scRNA-seq. The upper half of the body was collected at E8.5. The pharyngeal apparatus and heart were collected at E9.5. Sorted GFP-positive cells were used for scRNA-seq. Scale bar E8.5 is $400 \mu \mathrm{m}$; E9.5 is $500 \mu \mathrm{m}$. c Single-cell embedding graph of the CPM populations from the integration of two replicates of Mesp 7 Cre $\mathrm{Ctrl}$ vs $\mathrm{CKO}$ datasets colored by clusters. Definitions of clusters are the same as in Fig. 1, with

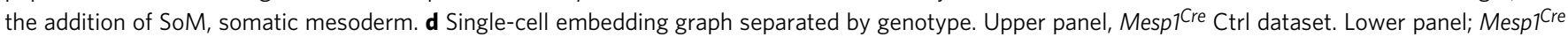
cKO dataset. e Single-cell embedding graph of integration of CPM lineages from Tbx7 Cre Ctrl and cKO datasets at E8.5 and E9.5 colored by cell clusters.

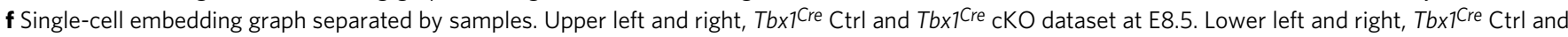
$\mathrm{Tbx}{ }^{\mathrm{Cre}}{ }_{\mathrm{CKO}}$ dataset at E9.5. $\mathbf{g}$, h Single-cell embedding graph of the CPM lineages from integrated Mesp ${ }^{\mathrm{Cre}} \mathrm{Ctrl}$ and cKO datasets (g) and Tbx ${ }^{\mathrm{Cre}} \mathrm{Ctrl}$ and cKO datasets (h) colored by expression level (gray to blue, green, yellow, low to high).

due to the presence of other cell populations, nevertheless, we found similar changes in MLPs with this independent comparison. Overall, the three independent scRNA-seq datasets suggest that $T b x 1$ promotes lineage maturation but restricts ectopic expression of non-mesodermal genes in MLPs.

In addition to MLPs, we examined DEGs in other CPM populations (BrM, SoM+aSHF, pSHF, and CMs; Supplementary Fig. 11 and Supplementary Data 9). Of interest, Tbx1 is expressed strongly in the BrM populations. We found that there are 667 genes decreased and 163 increased in expression in the BrM population at E9.5 (Supplementary Fig. 11a). Lhx2 and Myf5 are representative genes that are specific for and downregulated only in the BrM populations (Supplementary Data 9). This suggests the potential of direct transcriptional regulation. We also identified DEGs in other CPM-derivative populations, where Tbx1 is not strongly expressed (Supplementary Fig. 11), and examined GO 
a

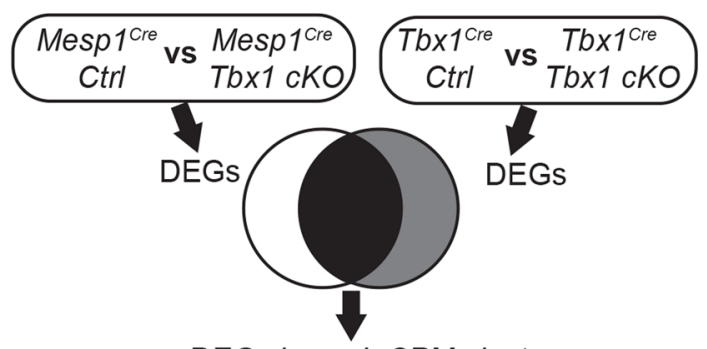

DEGs in each CPM cluster b

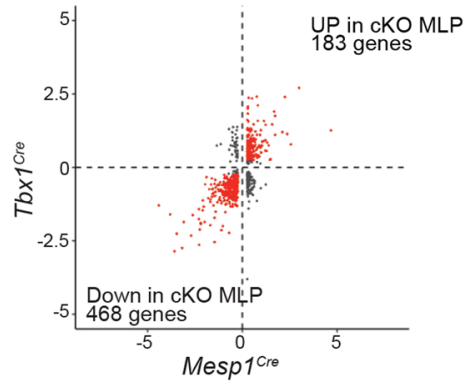

C

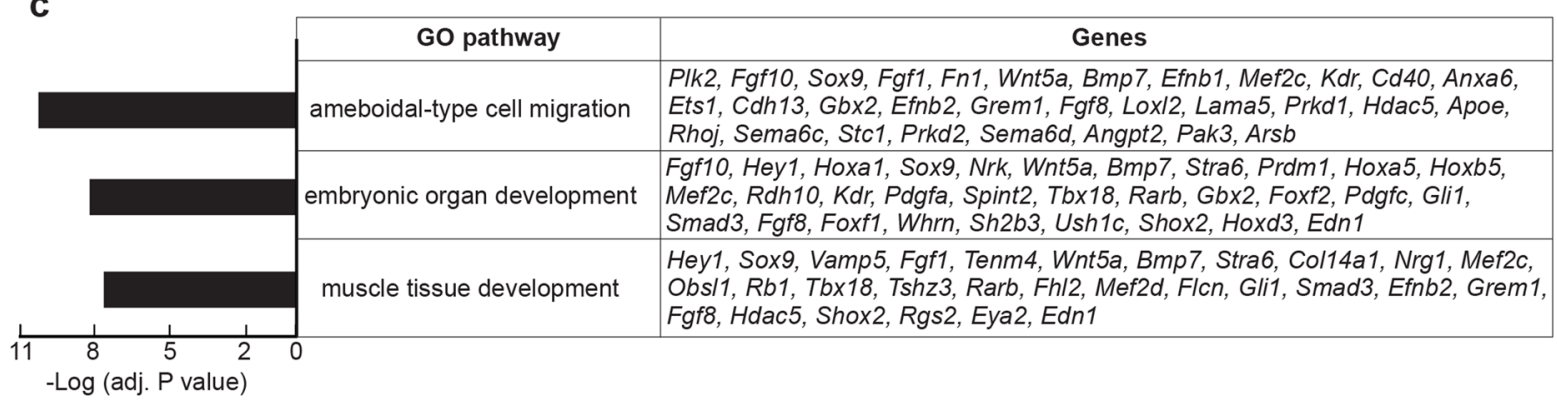

\begin{tabular}{|c|c|c|}
\hline \multirow[t]{4}{*}{ d } & GO pathway & Genes \\
\hline & axonogenesis & Tubb3, Gata3, Bdnf, Ncam1, Is/2, Nefl, Nefm, Nfib, Ntf3, Pax6, Unc5b, Rab21, Amigo1 \\
\hline & inner ear development & Pax8, Gata3, Bdnf, Lrig1, Mafb, Dvl3, Ror1, Itga8 \\
\hline & cell fate determination & Ebf2, Gata3, Ntf3, Pax6 \\
\hline
\end{tabular}

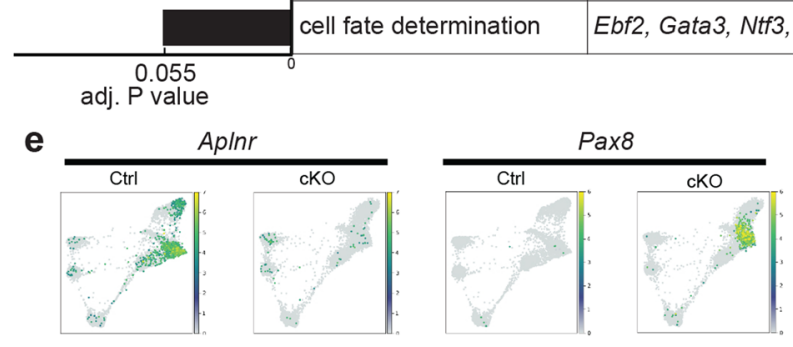

g

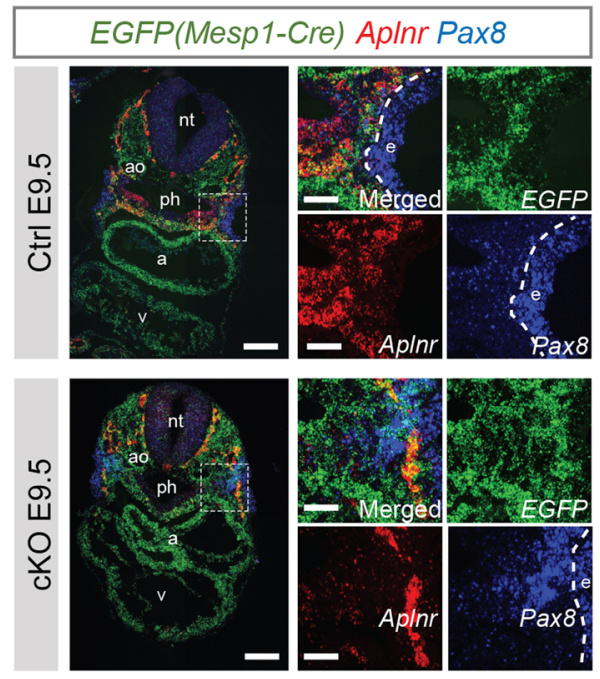

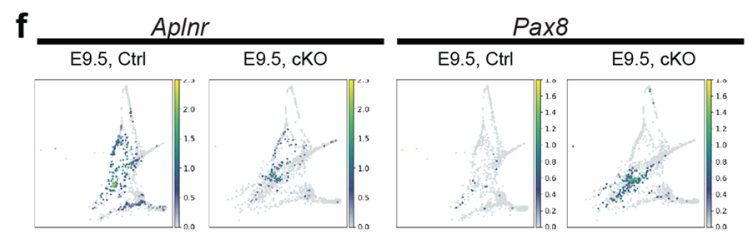

h

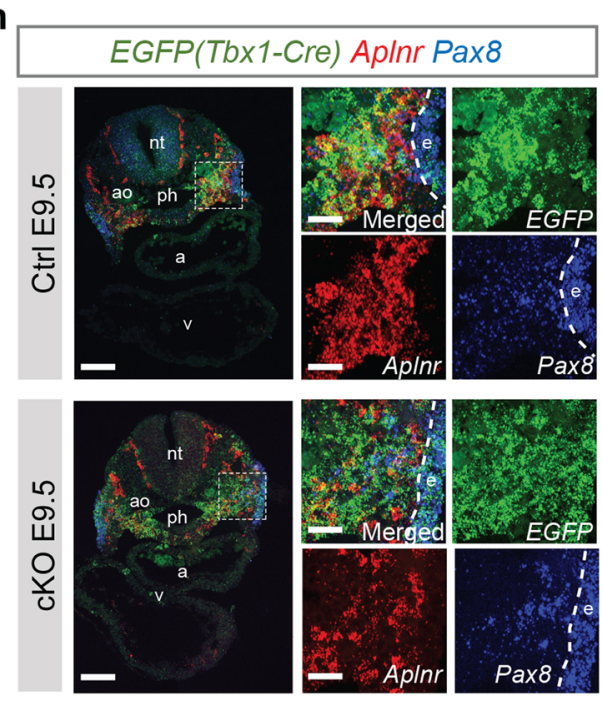

pathways affected (Supplementary Fig. 12 and Supplementary Data 10). We compared DEGs specific for MLP versus derivative CPM populations (Supplementary Fig. 13a). Representative DEGs are decreased in MLPs and derivative cells include Bmp7, Fgf10, and Foxf1, suggesting that these changes continue in derivative populations (Supplementary Fig. 13b-d). Other DEGs are reduced only in derivative CPM populations, even where $T b x 1$ is not strongly expressed (Supplementary Fig. 13a), suggesting indirect regulation and changes in cell fate acquisition.

TBX1 defines a gene regulatory network in the MLPs for cardiac and BrM formation. To better understand how TBX1 
Fig. 6 Tbx1 is required in MLPs to promote lineage progression and restrict the expression of ectopic genes. a The cartoon of analysis strategy focus on DEGs in the CPM that are shared between both lineages (black). b Comparison of differentially expressed genes (DEGs) from Mesp 7 Cre/t+; ROSA26-GFPf/+ (Ctrl) vs Mesp1Cre/+; ROSA26-GFPf/+;bxif/f (cKO) embryos at E9.5 and Tbx 1 Cre/+; ROSA26-GFPf/+ (Ctrl) vs Tbx 7 Cre/f; ROSA26-GFPf/+ (cKO) in MLPs. X axis indicates log2-fold change of Mesp1Cre DEGs. $Y$ axis indicates the log2-fold change of Tbx 7 Cre DEGs. Each dot indicates a gene, and red indicates common DEGs with |log2-fold change $\mid>0.25$ in both comparisons. c, d Enriched biological processes in gene ontology (GO) and related genes found in downregulated (c) or upregulated (d) genes in MLPs of Tbx1 cKO. $X$ axis indicates adjusted $P$ values. e Single-cell embedding graph of the CPM lineages from Mesp7 ${ }^{C r e} \mathrm{Ctrl}$ and CKO at E9.5 separated by genotype and colored by expression level (gray to blue, green, yellow, low to high). f Single-cell embedding graph of the CPM lineages from Tbx7 $7^{\text {re }} \mathrm{Ctrl}$ and CKO at E9.5 datasets separated by genotype and colored by expression level (gray to blue, green, yellow, low to high). $\mathbf{g}$ RNAscope in situ hybridization with EGFP (green), Ap/nr (red), and Pax8 (blue) mRNA probes on the transverse section from

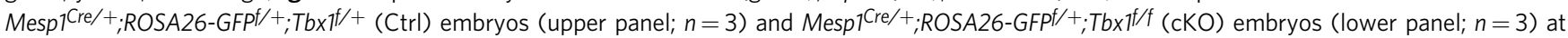
E9.5. Scale bar, $100 \mu \mathrm{m}$. The white dotted line indicates the position in higher magnification images shown in the right. Scale bar, $30 \mu \mathrm{m}$. $\mathbf{h}$ RNAscope in situ hybridization with EGFP (green), Aplnr (red), and Pax8 (blue) mRNA probes on the transverse section from Tbx 7 Cre/+;ROSA26-GFPf/+ (Ctrl) embryos (upper panel; $n=3$ ) and Tbxi Cre/f;ROSA26-GFPf/+ (cKO) embryos (lower panel; $n=3$ ) at E9.5. Scale bar, $100 \mu$ m. The white dotted line indicates the position in higher magnification images shown in the right (scale bar, $30 \mu \mathrm{m}$. a atrium, ao aorta, e ectoderm, nt neural tube, ph pharynx, v ventricle.

regulates the expression of genes in the $\mathrm{CPM}$ at the chromatin level, we used two biological replicates of ATAC-seq experiments of $T b x 1^{C r e} /+\left(T b x 1\right.$ Ctrl) versus $T b x 1^{C r e / f}$ (Tbxl cKO) mutant embryos (Supplementary Fig. 1c, d, Fig. 7a, Supplementary Fig. 14a-c, and Table 2). We chose stage E9.5 because this is when Tbx1 expression is the highest and when the OFT is elongating ${ }^{58}$. The ATAC-seq peaks were separated into commonly accessible regions (CARs) or differentially accessible regions (DARs; FDR < 0.05, Fig. 7b and Supplementary Fig. 14d) between Tbx1 Ctrl and Tbx1 cKO mutant samples. Among 5872 DARs, 5859 decreased and 13 increased in chromatin accessibility in Tbx $1 \mathrm{cKO}$ embryos (Supplementary Fig. 14d). To focus on the CPM, we next performed ATAC-seq on Mesp $1^{\mathrm{Cre} /+}$ cells in two biological replicates and used the peaks to exclude CARs and DARs that were found only in $T b x 1$ control and $T b x 1 \mathrm{cKO}$ samples, to eliminate changes in non-mesoderm lineages (Fig. $7 \mathrm{c}, \mathrm{d}$, gray regions). The remaining CARs and DARs are thus referred to as CARs-Mesp1 and DARs-Mesp1 (Fig. 7c, d). In DARs-Mesp1, several transcription factors binding motifs related to the heart or BrM differentiation including T-box motifs were enriched (Fig. 7e and Supplementary Data 11). More peaks in DARs-Mesp1 were found in distal intergenic regions than in CARs-Mesp1 (Supplementary Fig. 13e, f), suggesting that $T b x 1$ inactivation has a large effect on the regulation of genes through putative enhancer regions.

Next, we annotated DARs-Mesp1 (2185 peaks) to predicted target genes (2652 genes; Fig. $7 f$ and Supplementary Data 12). A total of 160 of the 468 DEGs that were downregulated (down in $\mathrm{cKO}$ ) in MLPs (Fig. 6b) were associated with DARs-Mesp1 (Fig. 7f, $P<1 \mathrm{e}-16$, chi-square test). GO analysis of the 160 genes indicated that they are involved in cell migration and muscle development, important for the function of Tbx1 (Fig. 7g). On the other hand, those without DARs-Mesp1 that were reduced in expression in mutant embryos were associated with the MAPK pathway and other functions (Fig. 7h). Of interest, Tbx1 might indirectly affect MAPK signaling in the MLPs that were not detected by ATAC-seq analysis and this is potentially mediated by well-known Tbx1-dependent FGF signaling 47,48 .

To determine which genes with DARs could be direct target genes of TBX1, we performed ChIP-seq with our new Avi-tagged Tbx1 mouse line and created double homozygous mice harboring the biotin ligase (BirA) gene $\left(T b x 1^{3^{\prime}-A v i} ;\right.$ BirA, Supplementary Fig. 15). We used input as background (sequencing depth replicate 1 is 26,318,633; replicate 2 is $29,821,823$; replicate 3 is $33,965,309$ ) to compare with peaks found by ChIP (sequencing depth replicate 1 is $28,070,687$; replicate 2 is $32,561,521$; replicate 3 is $32,907,512)$. We considered peaks found in at least two of the three replicates, each containing 20 embryos of 19-22 somites, with aligned peaks (replicate 1, 294; replicate 2, 128; replicate 3,536 peaks) as high confidence TBX1-binding sites
(Supplementary Fig. 16a-c). Out of 255 peaks, 176 peaks had a T-box motif (Fig. 8a, Supplementary Fig. 16d, e, and Supplementary Data 13), supporting TBX1 occupancy; 104 peaks (41\%) in the ChIP-seq data overlapped with DARs (Fig. 8a, b, $P<0.001$, permutation test). Comparing Tbx1 Ctrl and Tbx1 cKO ATACseq data in 255 ChIP-seq peak regions, we found that these regions were mostly open and accessible in Ctrl embryos but closed in Tbx $1 \mathrm{cKO}$ embryos (Fig. 8c and Supplementary Fig. 16f). Of the 255 ChIP-seq peaks, 151 (59\%) did not show significant chromatin accessibility changes (i.e., overlapping with DARs), which include some TBX1-binding sites in closed regions that did not change in accessibility in the mutant data (Fig. 8c), suggesting a diverse role of TBX1 in promoting chromatin remodeling.

ChIP-seq peaks were annotated to predicted direct transcriptional target genes (443 genes, 470 peaks, Supplementary Data 14). We then intersected the DEGs reduced in MLPs, the annotated genes from Mesp1-DARs and TBX1 ChIP-seq targets (Fig. 8b). We found 21 known genes (Fig. 8b and Supplementary Data 14) common in all three datasets $(P<0.001$; permutation test). Among them, eight had a DAR that overlapped with a TBX1 ChIP-seq peak (Slit1-intron, Crybg3-intron, Nrg1-upstream, Trps1-downstream, Sox9-upstream, Trmt9b-downstream, Fn1-upstream, and Crtc2promoter region). Data is consistent with TBX1 binding to accessible chromatin in control embryos, but the interval is not accessible when Tbx1 is inactivated. The rest had a DAR that did not overlap with a TBX1-binding site (Fgf1, Aplnr, Tshz3, Rcsd1, B3galnt12, Ppml1, Spon1, Mpped2, Tbx18, Daam1, Parvb); perhaps regulation is by long-range chromatin interaction with TBX1 binding. We show two representative examples, Aplnr and Nrg1, which are MLP enriched genes that are TBX1 direct transcriptional targets (Fig. 8d). In the Nrg1 locus, the TBX1-binding region was closed in Tbx1 cKO embryos. We examined the ENCODE ChIPseq tracks in the UCSC genome browser tracks ${ }^{49}$. For Nrgl, the colocalized peak is within an ENCODE cis-regulatory element (cCRE) that is a poised enhancer in mouse embryonic heart ${ }^{49}$. For the Aplnr gene region, the TBX1-binding site that is just downstream of the 3'UTR (Fig. 8d), is in an ENCODE cCRE that is a poised enhancer in mouse embryonic heart, but it was not in a DAR found in our data. The DAR that was identified in the Aplnr locus, is in an ENCODE cCRE (E0701748/enhD) and is an ATAC-seq peak region in the embryonic heart ${ }^{49}$. Overall, the regions found appear to be regulatory regions, but TBX1 might not always affect chromatin accessibility, indicating that multiple mechanisms of regulation occur.

Taking the results from the three types of functional genomic data in this report, we can generate a putative gene regulatory network for TBX1 function in the MLPs as summarized in Fig. 8e. Here, we distinguish four categories of genes potentially regulated by TBX1: (1) Direct target genes with or (2) without chromatin changes, and 
a

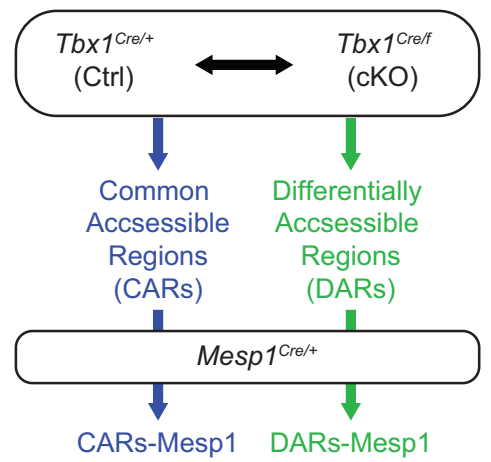

e DARs-Mesp1

\begin{tabular}{|c|c|c|c|c|c|}
\hline Rank & Motif & Transcription factor & $p$-value & $\begin{array}{l}\text { Back } \\
\text { ground }\end{array}$ & Targets \\
\hline \multirow{2}{*}{\multicolumn{2}{|c|}{ 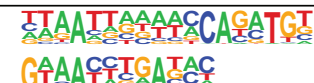 }} & Pitx1:Ebox(Homeobox,bHLH) & $1 \mathrm{e}-119$ & $0.88 \%$ & $8.70 \%$ \\
\hline \multirow{2}{*}{\multicolumn{2}{|c|}{ 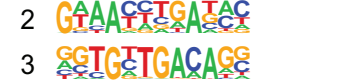 }} & Six2(Homeobox) & $1 e-80$ & $6.91 \%$ & $19.32 \%$ \\
\hline & & Tbx20(T-box) & $1 e-54$ & $2.18 \%$ & $8.61 \%$ \\
\hline \multicolumn{2}{|c|}{4 AGCCAATCGG } & NFY(CCAAT) & $1 \mathrm{e}-48$ & $8.18 \%$ & $18.04 \%$ \\
\hline \multicolumn{2}{|c|}{5 GGATCA } & Six1(Homeobox) & $1 e-47$ & $1.66 \%$ & $6.96 \%$ \\
\hline \multicolumn{2}{|c|}{6 AAAGTAAACA } & FOXA1(Forkhead) & $1 e-35$ & $6.71 \%$ & $14.38 \%$ \\
\hline \multicolumn{2}{|c|}{15 줄AㅕㄹCACCTGG } & Tcf21(bHLH) & $1 \mathrm{e}-27$ & $12.22 \%$ & $20.60 \%$ \\
\hline \multicolumn{2}{|c|}{66 TAAATTGG } & IsI1(Homeobox) & $1 e-13$ & $14.14 \%$ & $20.01 \%$ \\
\hline
\end{tabular}

f
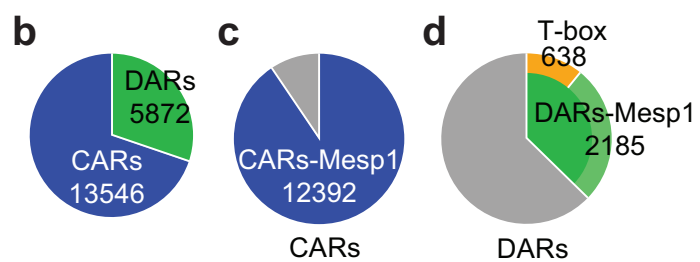

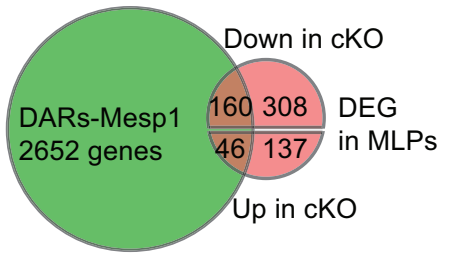

\section{DARs-Mesp1-DEGs down in cKO (160 genes)}

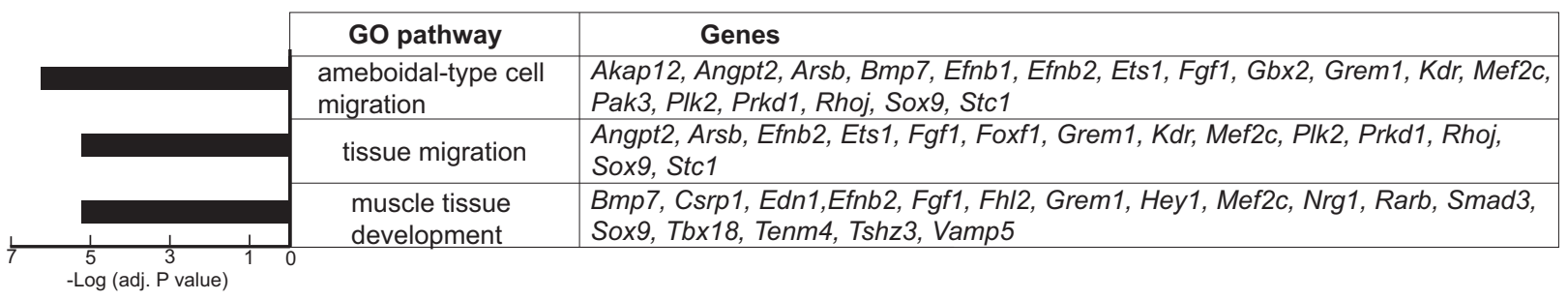

\section{h no DARs-Mesp1-DEGs down in cKO (308 genes)}

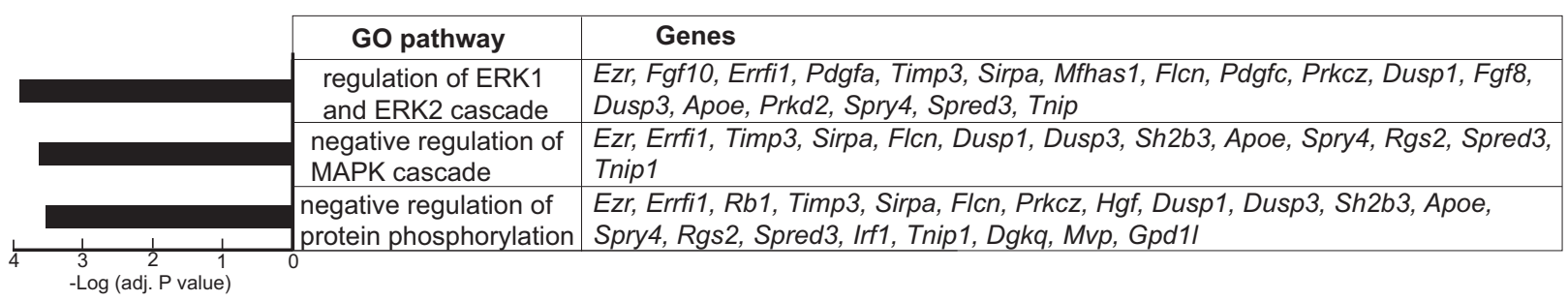

Fig. 7 Tbx1 affects gene expression and chromatin accessibility of MLPs. a Scheme of ATAC-seq data analysis. b Pie chart of ATAC-seq peaks of GFP + cells from Tbx1 Cre/t; ROSA26-GFPf/+ (Ctrl) and Tbx1 Cre/f; ROSA26-GFPf/+ (cKO) embryos at E9.5 $(n=3)$. A total of 13,546 peaks, defined as CARs, commonly accessible regions, were found in both $\mathrm{Tbx}_{7}{ }^{\mathrm{Cre}} \mathrm{Ctrl}$ and $\mathrm{cKO}$ datasets. A total of 5872 peaks, defined as DARs, differentially accessible regions, in which peak intensity was changed (FDR $\leq 0.05$ ) between $\mathrm{Tbx}{ }_{1} \mathrm{Cre} \mathrm{Ctrl}$ and $\mathrm{cKO}$ datasets. c Pie chart showing the intersection of CARs with ATAC-seq peaks of GFP + cells from Mesp7Cre/t; ROSA26-GFPf/+ embryos at E9.5 $(n=3)$. Among 13,546 CARs, a total of 12,392 peaks were also found to open in Mesp1 Cre lineages and thus referred to as CARs-Mesp1. d Pie chart showing the intersection of DARs with ATAC-seq peaks of GFP + cells from Mesp1Cre/ $+; R O S A 26-G F P f /+$ embryos at E9.5 $(n=3)$. Among 5872 DARs, 2185 were also found to open in Mesp 7 Cre lineages and thus referred as DARs-Mesp1. Of the DARs-Mesp1 regions, 638 had a T-box motif. e The motifs found in DARs-Mesp1 regions by Homer (hypergeometric optimization of motif enrichment) with default settings. $\mathbf{f}$ Venn diagram of genes with ATAC-seq DARs-Mesp1 regions and DEGs in MLPs from scRNA-seq. Of the 770 DEGs in the MLPs, 160 genes showed decreased ( $P$ value $<2.2 \mathrm{e}-16)$ and 46 genes showed increased were found as DARs-Mesp1 $(P$ value $<2.2 \mathrm{e}-16)$. $\mathbf{g}$ Enriched biological processes for the 160 genes with both DARs-Mesp1 and reduced expressed in Tbx1 cKO MLPs. $X$ axis indicates adjusted $P$ values. $\mathbf{h}$ Enriched biological processes for the 308 genes with reduced expression in Tbx1 cKO but without DARs-Mesp1. $X$ axis indicates adjusted $P$ values.

indirect target genes, (3) with chromatin changes, that contain transcription factor binding sites, and (4) without chromatin changes. Overall, we suggest that TBX1 with Isl1, Fox, Six, Pitx, and E-box proteins such as Tcf21, (Fig. 8e), act together to regulate the progression of MLPs to more differentiated states in the CPM.

\section{Discussion}

In this report, we discovered a progenitor cell population within the CPM that we term MLP. We discovered that MLPs are localized bilaterally within the posterior nascent mesoderm of the pharyngeal apparatus. The MLPs function to both maintain a 
Table 2 Summary of ATAC-seq samples.

\begin{tabular}{|c|c|c|c|c|}
\hline & $\begin{array}{l}\text { No. of } \\
\text { embryos }\end{array}$ & Somites & $\begin{array}{l}\text { Cell number } \\
\text { GFP( }+ \text { ) }\end{array}$ & Aligned peaks \\
\hline \multicolumn{5}{|c|}{ Mesp1-Cre; GFPf/+ } \\
\hline E9.5-1 & 3 & $20-21$ & 10,000 & 25,485 \\
\hline E9.5-2 & 2 & $19-21$ & 10,000 & 30,535 \\
\hline E9.5-3 & 5 & $21-22$ & 10,000 & 21,161 \\
\hline \multicolumn{5}{|c|}{ Tbx1-Cre;GFPf/+ } \\
\hline E9.5-1 & 5 & $19-22$ & 10,000 & 49,490 \\
\hline E9.5-2 & 5 & $19-21$ & 10,000 & 43,298 \\
\hline E9.5-3 & 5 & $19-21$ & 10,000 & 47,013 \\
\hline \multicolumn{5}{|c|}{ Tbx1-Cre/f; GFPf/+ } \\
\hline E9.5-1 & 4 & $19-22$ & 10,000 & 29,251 \\
\hline E9.5-2 & 3 & $21-22$ & 10,000 & 24,042 \\
\hline E9.5-3 & 2 & 21 & 10,000 & 19,772 \\
\hline
\end{tabular}

progenitor state and promote differentiation to derivative cell types as shown in the model in Fig. $8 \mathrm{f}$. Tbx1 is required for the MLPs to express critical genes for cardiac and BrM development, and to prevent ectopic expression of non-mesodermal genes, needed for maturation of the CPM-derivative cells (Fig. 8f). This is mediated by direct and indirect transcriptional regulation and chromatin accessibility allowing for cell state progression.

Previous work showed that the aSHF, pSHF, and BrM cells comprising the CPM, derive from a relatively small number of Mesp1 expressing progenitor cells at gastrulation ${ }^{8}$. Based upon the work presented in this report, it is most likely that not all cells have committed to final CPM fates at gastrulation and MLPs provide a source of progenitors as the pharyngeal arches form. Retrospective clonal analysis has shown that there is a direct clonal relationship between progenitor cells that form the muscles of mastication and right ventricle, which are derived from the first pharyngeal arch, with distinct clones that forms both the OFT and facial expression muscles, from the second arch, while separate clones form the neck muscles and venous pole of the caudal pharyngeal arches, 3-69,30. This suggests that different clones contribute to different arches. It is possible that MLPs are comprised of heterogenous progenitor cells that are allotted to individual arches and/or that they are exposed to different extracellular signals during development, conferring pharyngeal arch-specific fates.

The heart tube elongates from E8 to 10.5 by the deployment of progenitor cells to the OFT. It is known that cells deployed to the cardiac poles first arrive at the dorsal pericardial wall (DPW), just behind the heart tube ${ }^{50}$. Mesodermal cells lateral and behind the DPW are thought to be incorporated to the DPW and then to the poles of the heart ${ }^{51}$. The deployment of mesoderm cells to the DPW provides a pushing force as the epithelial transitioned cells move to the poles of the heart ${ }^{51,52}$. In Tbx1 null mutant embryos, there are fewer cells in the DPW resulting in a shortened cardiac $\mathrm{OFT}^{35,43,53}$. We propose that MLPs comprise the dorsal population of mesoderm progenitor cells that are needed to allocate cells to the DPW. This is consistent with the reduction of Wnt5a expression in our data, as $W n t 5 a$ is a key downstream gene of $T b x 1$, required for their deployment ${ }^{51,52}$.

An anterior-posterior border is established in the DPW between $T b \times 1$ and Tbx5 expressing cells, respectively, that provides cells to the poles of the heart ${ }^{34}$. Consistent with this, we found that there are few $T b x 5$ expressing cells in the $T b x 1$ lineage. Understanding the molecular mechanisms of how the anterior-posterior border is established is an active area of research $^{5,34}$. We previously found that global inactivation of $T b \times 1$ results in increased expression of caudal pSHF genes such as Tbx5 and cardiac muscle genes 20,54 . Data presented in this report and recently ${ }^{34}$, indicate that rather than changes in expression, there are instead cell population changes in Tbx1 mutant embryos. Therefore, this scRNA-seq study discerns better between expression versus population changes depending on $T b x 1$, which is often a key challenge in interpreting developmental phenotypes. More work needs to be done in the future to better understand how these borders are formed and maintained.

In addition to deploying cells to the DPW and then to the heart, the MLPs express genes required for BrM formation in each arch. The BrMs form segmentally in a rostral to the caudal manner, in which the muscles of mastication form first from the first arch and the other muscles of the face and neck form thereafter from more caudal arches. The BrMs express myogenic regulatory transcription factors, including $T c f 21, M s c, M y f 5$, and later $M y o d 1^{55}$. In addition, transcription factor genes such as $I s l 1^{11}$, Pitx $2^{56}, T b x 1^{19}$, Lh $x 2^{22}$, and Ebf genes $\left(\right.$ Ciona ${ }^{57}$ ) are expressed in the CPM and are required for BrM formation. A subset of MLPs expressing Tbx1 will later express BrM genes as they migrate to the core of the pharyngeal arches. These cells progressively express myogenic transcription factor genes as they become restricted to form BrM skeletal muscle cells. Besides the MLPs, Tbx1 is also expressed in BrM progenitor cells, and therefore, some of the gene expression changes we observed in these cells might be due to Tbx1 expression in the BrM progenitor cells themselves.

Although we focus on the CPM as it relates to cardiac and skeletal muscle development, it was shown that the CPM also contributes to mesenchyme of connective tissue, including cartilage in the neck ${ }^{58}$. Thus, it is possible that the MLPs could promote connective tissue fates in the craniofacial region that are dependent on Tbx1. Further work will be needed to assess their lineage relationships. Given that most patients with 22q11.2DS have craniofacial malformations in the face and neck, it is important to understand the developmental trajectories of Tbx1dependent connective tissue progenitor cells.

A strength of this study is that we used two different Cre lines $\left(M e s p 1^{C r e}\right.$ nor $\left.T b x 1^{C r e}\right)$ and analyzed genes altered in both lines. This is because neither Mesp1 $1^{\text {Cre }}$ nor $T b x 1^{\text {Cre }}$ is sufficient to define the CPM, and further, both label non-relevant cell lineages. Since the cell number isolated from these embryos was relatively small, it is also possible that rare populations would be missed, so that using this strategy reduces the concern of under sampling. Further, there are limitations of using the $\mathrm{Tb} \times 1^{\mathrm{Cre}}$ allele to compare with gene expression changes in Tbx1 ${ }^{\text {Cre }}$ conditional null mutant embryos. In $T b \times 1^{C r e /+}$ control embryos, one copy of Tbx 1 is inactivated. However, we did not observe cardiac defects in $T b x 1^{C r e}$ heterozygous embryos or $T b x 1^{+/-}$embryos in which a different region of $T b x 1$ was inactivated, as maintained in the SwissWebster background. This is in comparison to Tbx1 heterozygous mice as maintained in $\mathrm{C} 57 \mathrm{Bl} / 6$, in which heterozygous mice have pharyngeal arch artery defects at reduced penetrance ${ }^{16-18}$. This suggests that the mild phenotype observed is not due to localized genetic modifiers in the Tbx1 locus itself but rather the genetic background.

The effect of genetic background upon the pharyngeal arch artery phenotype of $T b \times 1$ heterozygous mice has been known for many years. Early work noted that the original 129 strain, in which the Tbx $1^{C r e}, T b x 1^{f /+}$, and $T b x 1^{+/-}$mutations were generated, has a suppressive effect upon the penetrance of such defects (similarly to SwissWebster), while the C57Bl/6 background has an enhancing effect, and research excluded that this modifying effect was due to modifications in the localized genomic region ${ }^{59}$. Further, we have not observed the presence of novel or unusual transcripts in the Tbx1 locus in different alleles in our scRNA-seq data. Finally, this work uses the null phenotype to draw conclusions about the role of Tbx1 in development, not the 
a

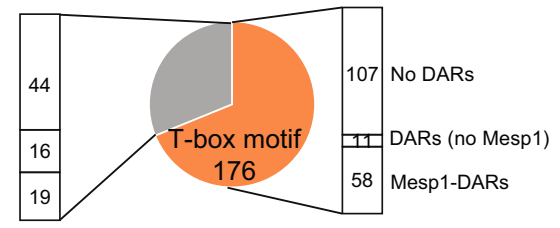

b

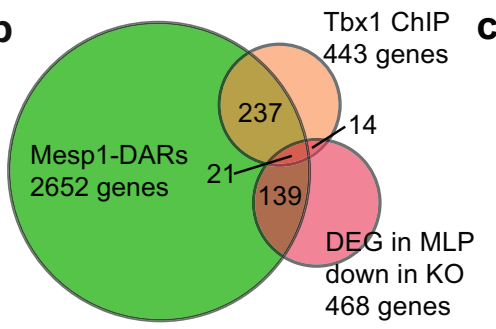

d

Chr 8genomic interval Tbx1-ChIP ATAC $\mid \begin{aligned} & \text { Tbx1+ } \\ & \text { Tbx1+cKO } \\ & \text { Mesp1+ }\end{aligned}$ Genes

\section{$\operatorname{Nrg} 1$}

.

Chr 2genomic interval

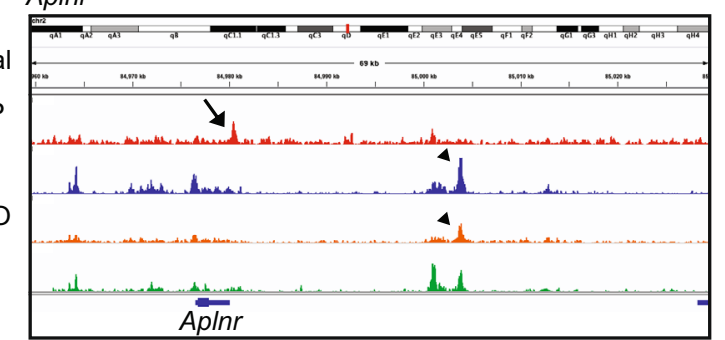
Genes

e

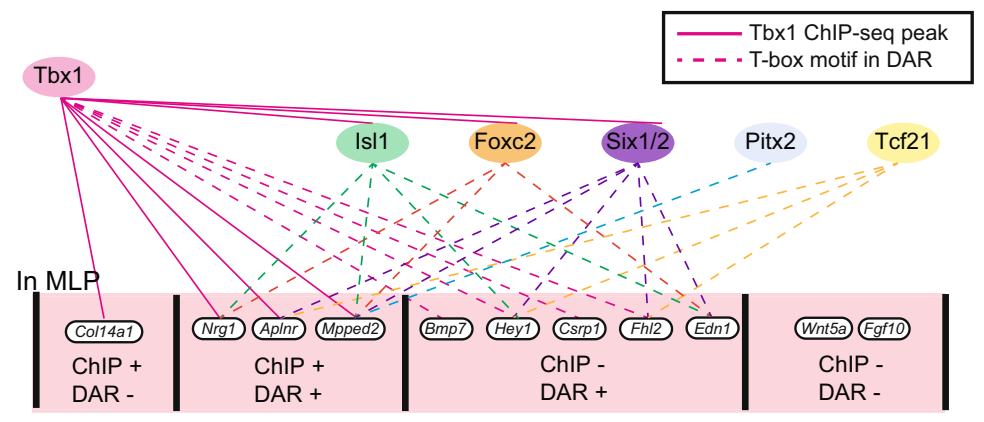

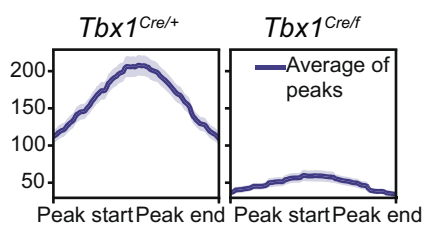

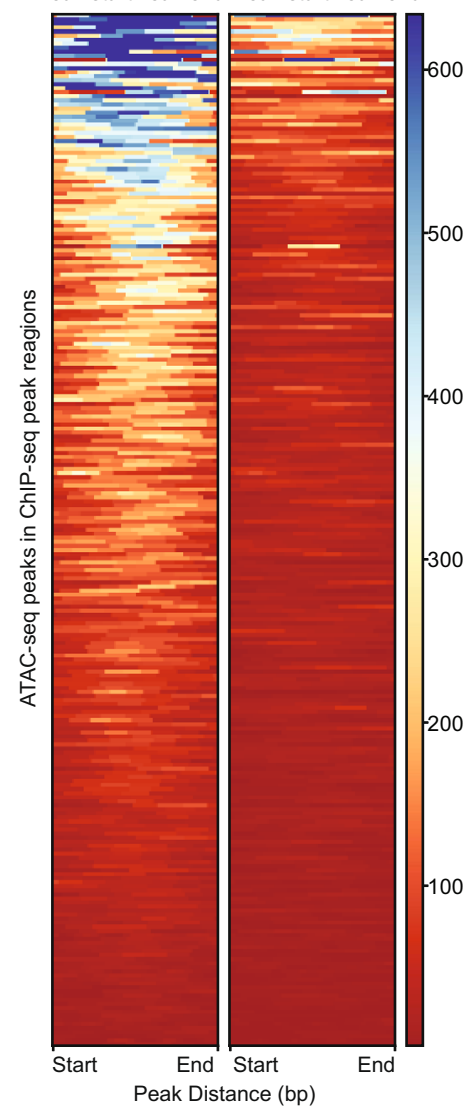

f

Control

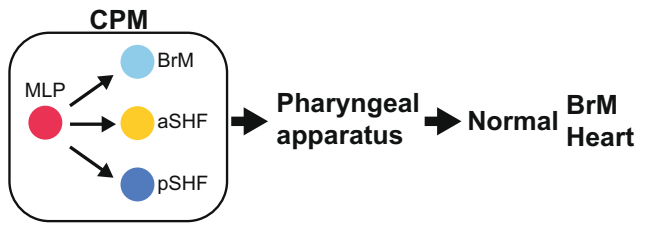

heterozygous phenotype. The null phenotype, as two decades of publications indicate, is remarkably similar across multiple genetic backgrounds. Therefore, the genetic backgrounddependence of the heterozygous phenotype would not affect the major conclusions of the manuscript.

Although there are likely some gene expression changes in $\mathrm{Tb} \times 1^{\mathrm{Cre} /+}$ embryos versus wild-type embryos, we obtained
Tbx1 deficient

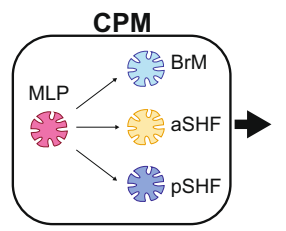

Failed CPM

maturation Hypoplastic/missing BrM

Hypoplasia of 3-6 arches 
Fig. 8 Open chromatin status in TBX1-binding regions in vivo was reduced in Tbx1 cKO embryos. a Pie chart of TBX1 ChIP-seq peaks from three biological replicates. Among $255 \mathrm{ChIP}$-seq peaks, 176 had a T-box motif. The bar chart indicates peak intersection with ATAC-seq data. b Venn diagram for overlap of genes with ChIP-seq peaks or ATAC-seq DARs-Mesp1 regions or downregulated expression in Tbx1 cKO MLPs $(P$ value $=0.3924)$. c Average chromatin accessibility at ChIP-seq peaks. Left, ATAC-seq data from Tbx ${ }^{C}$ re $\mathrm{Ctrl}$; right, ATAC-seq data from Tbx 7 Cre cKO. Top, average ATAC-seq signal in TBX1 ChIP-seq peak regions; Bottom, heatmap of ATAC-seq read densities in TBX1 ChIP-seq peaks. Statistical significance (adj. P<0.0009) of ChIP-seq peaks on ATAC-seq signal has been tested using enrichPeakOverlap present in ChIPseeker. d Genome browser snapshots including results from TBX1 ChIP-seq, ATAC-seq of Tbx 7 Cre Ctrl, Tbx 7 Cre cKO, and Mesp Cre lineages, at Nrg1 (upper), Aplnr (lower) loci. Arrow indicates TBX1 ChIP-seq peak. Arrowhead indicates DARs. e A cartoon of the TBX1 regulation network in the MLPs and derivatives. Pink lines indicate TBX1 direct target genes from in vivo ChIP-seq. Dotted lines indicate genes with the TBX1 motif harboring DARs-Mesp1 from ATAC-seq analysis. The transcription factor genes indicated in different colors (Is/1, Foxc2, Six1/2, Pitx2, Tcf21) are representative family members sharing the same binding motif enriched in DARs-Mesp1. f A working hypothesis of MLP function with respect to Tbx1. In normal embryogenesis, MLPs provide progenitors that can form cardiac and BrM skeletal muscle. In Tbx1 conditional null embryos, the cellular state of MLPs is disrupted by the loss of direct and indirect transcriptional regulation (shown as flower shape). This leads to failed formation of CPM derivatives, which causes BrM and cardiac OFT defects.

To understand the molecular mechanisms of Tbxl function we performed ATAC-seq and TBX1 ChIP-seq. We generated a gene regulatory pathway downstream of $T b x 1$ in MLPs that is required for the maturation of cells to CPM derivatives. We obtained robust data from the ATAC-seq experiments and found alteration of chromatin accessibility when Tbx1 was inactivated, of which some harbored putative T-box-binding sites. The TBX1 ChIP-seq provided hundreds of direct target genes, of which some are also reduced in expression in mutant embryos and show a change in chromatin accessibility. One note is that we identified hundreds rather than thousands of direct transcriptional targets that were expected based upon studies of other transcription factors. This could be because we used whole embryos for the ChIP-seq with lower tissue yield than microdissection. Nonetheless, the ChIPseq data supports the ATAC-seq findings. Further, this work shows that both direct and indirect regulation occurs downstream from TBX1 because not all differentially accessible sites have Tsites, and not all differentially expressed genes have either differentially accessible sites or ChIP-seq peaks. Additionally, TBX1 protein can regulate the protein level of serum response factor, SRF without changing the expression level of RNA, thus there are possible other functions of TBX1 that do not involve binding to $\mathrm{DNA}^{22}$. We identified MAPK pathway genes are altered indirectly downstream of $T b x 1$, suggesting that this is a result of the alteration of well-known Tbx1-dependent FGF signaling ${ }^{47,48}$. A subset of genes found with differentially accessible regions in mutant embryos was expressed in MLPs as well as derivative cell types. This is particularly true for the BrM progenitor cells because this is where $T b x 1$ is actively expressed.

By performing these multi-omic studies, we identified Aplnr and $\mathrm{Nrg} 1$ among the genes enriched in expression in MLPs. Aplnr encodes the APJ/Apelin G-protein coupled receptor that binds Apelin or Elabela/Toddler peptide ligands that have many embryonic and adult functions ${ }^{60}$. In zebrafish, knockdown of Aplnr disrupts normal migration of cells during gastrulation including that of cardiac progenitors resulting in severe defects ${ }^{61,62}$. Unexpectedly, their role in early embryogenesis is not recapitulated fully in mouse models, implicating perhaps functional redundancy with other G-protein-coupled receptors or ligands ${ }^{63}$. Aplnr is expressed in the CPM, and from stem cell studies, has a role in cardiomyocyte development ${ }^{27}$. Nrg1 is also of particular interest. In contrast to Aplnr, Nrg1 is not expressed in the DPW. Nrg1 encodes an EGF family ligand that binds to ErbB receptor tyrosine kinases and has multiple roles in cardiac development and function ${ }^{28,64}$. Interestingly, both Aplnr and Nrg1 are direct target genes of TBX1 based on our ATAC-seq and ChIP-seq results, however, more work will need to be done to know whether these genes have functional importance in MLPs or in relation to Tbx1.

Among the genes that were differentially expressed and differentially accessible in Tbxl conditional null embryos are
Isl1 $1^{20,65}$, Foxc $2^{66,67}$ Six1/Six $2^{48}$, Pitx $2^{56,68}$, and Tcf2 $1^{22}$. Isl1, Foxc2, and Six2 may be direct transcriptional target genes of TBX1 based upon ChIP-seq analysis. Some known downstream genes of Tbxl were not identified in the multi-omic data, such as Wnt5a ${ }^{69}, F g f 10^{70,71}$, and $N k \times 2-5$, possibly due to low transcript abundance, incomplete set of TBX1 target genes from ChIP-seq, or non-autonomous functions in neighboring CPM cells.

The distal pharyngeal apparatus is hypoplastic when $T b \times 1$ is inactivated in the mesoderm ${ }^{72}$. This is in part because the loss of Tbx1 severely affects pharyngeal endoderm-mediated segmentation ${ }^{36}$ affecting neighboring neural crest cell populations ${ }^{72}$. These functions are non-autonomous between the CPM and neural crest cells, given that $T b x 1$ is not expressed in neural crest cells that contribute to OFT septation ${ }^{73}$. It is possible that altered signaling from affected pharyngeal endoderm cells or lack of neural crest cells could influence MLP or CPM differentiation, besides cell or tissue autonomous effects.

Global inactivation of $T b x 1$ results in a persistent truncus arteriosus and hypoplastic muscles of mastication and failure to form the facial/neck muscles ${ }^{16,19,74}$. Here, we report the existence of a mesodermal cell population termed MLPs. We show that inactivation of Tbx1 results in dysregulation of gene expression in MLPs affecting their cellular state. Once this occurs, differentiation is affected as well as signaling to adjacent cells thereby altering migration and/or survival of derivative CPM cells leading to the observed phenotypes. The MLPs are thus a continuous but evolving source of CPM cells that is maintained during the development of the pharyngeal apparatus and plays a critical role in craniofacial and cardiac development as well as pathology.

\section{Methods}

Mice. All experiments using mice were carried out according to regulatory standards defined by the National Institutes of Health and the Institute for Animal Studies, Albert Einstein College of Medicine (https://www.einsteinmed.org/ administration/animal-studies/), IACUC protocol is \#0000-1034.

The following mouse mutant alleles used in this study are: Mesp $1^{\mathrm{Cre} 7}, \mathrm{~Tb} \times 1^{\mathrm{Cre} 75}$ Tbx $1^{f / f 76}, T b x 1^{+/-76}$, ROSA26-GFPf/f $(\text { RCE: loxP })^{77}$. Tbx $1^{C r e /+}$, Tbx $1^{f /+}$, Mesp $1^{\text {Cre/++, }}$ and ROSA26-GFPf/+, single heterozygous mice have been backcrossed in SwissWebster strain for over 15 generations. The Tbxif/f, ROSA26-GFPf/f, and ROSA26-GFPf/f; $T b x 1^{f / f}$ homozygous mice were inter-crossed in brother $\times$ sister crosses for over 20 generations and constitute inbred lines. The breeding strategies for all the experiments are illustrated in Supplementary Fig. 1. For lineage tracing in Tbx1 conditional knockout embryos, ROSA26-GFPf/f; Tbx $1^{f / f}$ and ROSA26-GFPf $f ; T b \times 1^{f /+}$ mice were generated by inter-crossing Tbx $1^{f / f}$ and ROSA26-GFPf/f mice. To generate $M e s p 1^{C r e /+} ; R_{O S A 26-G F P f /+}$ embryos, Mesp1 ${ }^{C r e /+}$ male mice were crossed with ROSA26-GFPf/f female mice (Supplementary Fig. 1a). To generate

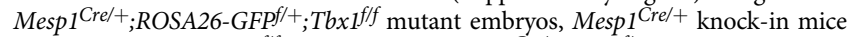
were crossed with $T b x 1^{f / f}$ mice to obtain Mesp $1^{C r e /+} ; T b x 1^{f /+}$ male mice, then crossed with ROSA26-GFPf/f;Tbx1 f/f female mice (Supplementary Fig. 1b). The littermates with Mesp1 $1^{\mathrm{Cre} /+} ;$ ROSA26-GFPf/+ $T b \times 1^{f /+}$ were used as the Ctrl in RNAscope experiments. To generate Tbx1 ${ }^{\mathrm{Cre} /+} ;$ ROSA26-GFPf/+ and Tbx1 ${ }^{\mathrm{Cre} /}$

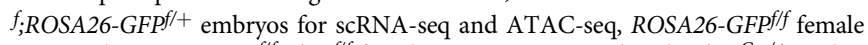
mice and ROSA26-GFPf/f; $\mathrm{Tb} \times 1^{f / f}$ female mice were crossed with $\mathrm{Tb} \times 1^{\mathrm{Cre} /+}$ male mice, respectively (Supplementary Fig. 1c, d). For RNAscope analysis, littermates were used from a cross of $T b x 1^{C r e /+}$ male mice and ROSA26-GFPf/f; $T b x 1^{f /+}$; female 
mice (Supplementary Fig. 1e). All the mice are maintained on the SwissWebster genetic background. The PCR strategies for mouse genotyping have been described in the original papers and are available upon request.

To generate $T b x 1^{3^{3}-A v i}$ mice, Avidin (Avi) was inserted using CRISPR/Cas9 genomic engineering in the Gene Modification Facility of Albert Einstein College of Medicine. A guide RNA (gRNA) targeting to the 3' last exon of Tbx1 (5'gcgcgcgggcgcactatctgggg- $3^{\prime}$ ) was designed by Guide Design Resources (http:// crispr.mit.edu/) and generated by in vitro transcription ${ }^{77}$. Cas9 mRNA was purchased from SBI. Tbx $1^{3^{3}-A v i}$ homology directed repair (HDR_vector containing the $60 \mathrm{nt}$ homologous arms (5'-ggcggccgcgecgcccggtgcctacgactactgccccagaGGTGG AAGTggcctgaacgacatcttcgaggctcagaaaatcgaatggcacgaatagtgcgcccgcgcgccgaccccgagg gccatccaaggacgcgctccc- $3^{\prime}$ ) at each side surrounding the Avi tag was synthesized chemically from IDT. Super-ovulated female C57BL6 mice (3-4 weeks old) were crossed with $\mathrm{C} 57 \mathrm{BL} / 6$ males, and fertilized embryos were collected from oviducts. The gRNA, Cas9 mRNA and Tbx 1 $^{3^{\prime}-A v i}$ HRD vectors were mixed and microinjected into the cytoplasm of fertilized eggs. The injected zygotes were transferred into pseudopregnant $\mathrm{CD} 1$ females, and the resulting pups were obtained. For genotyping, $311 \mathrm{bp}$ (wild-type) and $365 \mathrm{bp}\left(\mathrm{Tbx} \mathrm{1}^{3}\right.$-Avi $)$ bands were

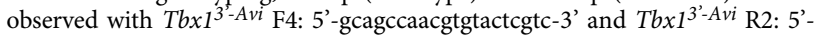
gccggtgcagtatctacagt-3' primer pair. After several backcrosses with Swiss Webster mice, Tbx $1^{3^{\prime}-A v i}$ mice were crossed with FVB;129P2-Gt(ROSA)26Sor ${ }^{t m 1.1(B i r A) M e j r} / J$ (BirA) mice from the Jackson laboratory to obtain Tbx $1^{3^{\prime}-A v i} ;$ BirA double homozygous mice.

The embryos at E8.0-E10.5 determined by standard somite counts, were used for all the experiments. We did not genotype for sex and we used both male and female embryos for all the experiments.

scRNA-seq. The embryos at E8.0-10.5 were isolated and GFP-positive embryos were selected under a SteREO Discovery.V12 microscope (Carl Zeiss, Jena, Germany) in ice-cold DPBS with $\mathrm{Ca}^{2+}$ and $\mathrm{Mg}^{2+}$ (GIBCO, Cat\# 14040-133). The rostral half of the embryos were collected at E8.0, E8.25, and E8.5. The pharyngeal apparatus with heart was collected at E9.5 and E10.5, as shown in Fig. 1b. The microdissected tissues were kept on ice and pooled in DMEM $\left(4^{\circ} \mathrm{C}\right.$, GIBCO, Cat $\#$ $11885-084)$ until all the dissections were completed. Following centrifugation $\left(4^{\circ} \mathrm{C}\right.$, $100 \times g$, 5'; ' is minutes) and removal of DMEM, tissues were incubated with $0.25 \%$ Trypsin-EDTA (GIBCO, Cat\# 25200-056) with Dnase I (50 U/ml) (Millipore, Cat\# 260913-10MU), 10' at RT. Then FBS (heat-inactivated, ATCC, Cat\# 30-2021) was added to stop the reaction. After centrifugation $\left(4^{\circ} \mathrm{C}, 300 \times g, 5^{\prime}\right)$, the cells were resuspended in PBS w/o $\mathrm{Ca}^{2+}$ and $\mathrm{Mg}^{2+}$ (Corning, Cat\# 21-031-cv) with 10\% FBS and passed through the $100-\mu \mathrm{m}$ cell strainer. DAPI (Thermo Fisher Scientific, Cat\# D3571) was added before cell sorting. The GFP + DAPI - cells were sorted with the BD FACSAria II system (Becton, Dickinson Biosciences, Franklin Lakes, NJ) with BD FACSDiva 8.0.1 software (Becton, Dickinson Biosciences). The sorted GFP-positive cells were centrifuged $\left(4^{\circ} \mathrm{C}, 300 \times g, 5^{\prime}\right)$, and resuspended in $50 \mu \mathrm{l}$ PBS w/o $\mathrm{Ca}^{2+}$ and $\mathrm{Mg}^{2+}$ with $10 \%$ FBS. After measuring cell number and cell viability, the cells were loaded in a 10x Chromium instrument (10x Genomics, Pleasanton, CA) using Chromium Single Cell 3' Library \& Gel Bead Kit v2 (10x Genomics, Cat\# PN-120237) or Chromium Next GEM Single Cell 3' GEM, Library \& Gel Bead Kit v3.1 (10x Genomics, Cat\# PN-1000121) according to the manufacturer's instructions (Genomics Core Facility). The concentrations of the libraries were measured with Qubit ${ }^{\circledR}$ 2.0 Fluorometer (Thermo Fisher Scientific, Waltham, MA) using Qubit dsDNA HS Assay Kit (Thermo Fisher Scientific, Cat\# Q32851). The details of each sample are provided in Table 1.

ATAC-seq. The ATAC-seq method has been previously described ${ }^{78}$; however, we provide details in this section. The embryos at $\mathrm{E} 9.5$ were isolated from a euthanized mother. GFP-positive embryos were selected under a SteREO Discovery.V12 microscope in ice-cold DPBS with $\mathrm{Ca}^{2+}$ and $\mathrm{Mg}^{2+}$ (GIBCO, Cat\# 14040-133). The pharyngeal apparatus without the heart was collected and pooled in DMEM (GIBCO, Cat\# 11885-084) until all the dissections were completed. Following centrifugation $\left(4^{\circ} \mathrm{C}, 100 \times g, 5^{\prime}\right)$ and removal of DMEM, tissues were incubated with $0.25 \%$ Trypsin-EDTA (GIBCO, Cat\# 25200-056) with Dnase I (50 U/ml) (Millipore, Cat\# 260913-10MU), 10' at RT. Then FBS (ATCC, Cat\# 30-2021) was added to stop the reaction. After centrifugation $\left(4^{\circ} \mathrm{C}, 300 \times g, 5^{\prime}\right)$, the cells were resuspended in PBS w/o $\mathrm{Ca}^{2+}$ and $\mathrm{Mg}^{2+}$ (Corning, Cat\# 21-031-cv) with 3\% FBS and passed through the 100- $\mu \mathrm{m}$ cell strainer. DAPI (Thermo Fisher Scientific, Cat\# D3571) was added before the cell sorting. The GFP + DAPI- cells were sorted with the BD FACSAria II system (Becton, Dickinson and Company, Franklin Lakes, NJ). The sorted cells were centrifuged $\left(4^{\circ} \mathrm{C}, 800 \times g, 5^{\prime}\right)$, and washed with PBS w/o Ca ${ }^{2+}$ and $\mathrm{Mg}^{2+}$. After centrifugation $\left(4^{\circ} \mathrm{C}, 800 \times g, 5^{\prime}\right)$, cells were resuspended with icecold Lysis buffer which contained $10 \mathrm{mM}$ Tris-HCl pH 7.4 (Thermo Fisher Scientific, Cat\# BP152-1), $10 \mathrm{mM} \mathrm{NaCl}$ (Thermo Fisher Scientific, Cat\# AM9760G), $3 \mathrm{mM} \mathrm{MgCl} 2$ (MilliporeSigma, Cat\# M9272) and 0.1\% Igepal CA-630 (MilliporeSigma, Cat\# I8896). Following centrifugation $\left(4^{\circ} \mathrm{C}, 1000 \times g, 5^{\prime}\right)$, nuclear pellets were incubated with Tn 5 transpose from the Nextera DNA Sample Preparation Kit (Illumina, Cat\# FC-121-1030) for $37^{\circ} \mathrm{C}, 30^{\prime}$. Transposed DNA was purified using the MinElute PCR purification kit (QIAGEN, Cat\# 28004), according to the manufacturer's instructions. The transposed DNA was amplified with NEB Net High Fidelity $2 \times$ PCR master mix, PCR primer cocktail, and two index primers from the Nextera Index Kit (Illumina, Cat\# FC-121-1011). PCR conditions for amplification is one cycle $5^{\prime} 72^{\circ} \mathrm{C}, 30^{\prime \prime}$ (seconds) $98^{\circ} \mathrm{C}, 12$ cycle $10^{\prime \prime}$ $98^{\circ} \mathrm{C}, 30^{\prime \prime}, 63^{\circ} \mathrm{C}, 1^{\prime}, 72^{\circ} \mathrm{C}$. PCR products were purified using the MinElute PCR purification kit. DNA concentration was measured with a Qubit ${ }^{\oplus 2.0}$ Fluorometer using Qubit dsDNA HS Assay Kit. DNA qualities were analyzed with a Bioanalyzer (Agilent Technologies, Santa Clara, CA).

ChIP-seq. Whole embryos from $T b x 1^{3}$-Avi;BirA double homozygous embryos or BirA homozygous embryos (Ctrl) at E9.5 were collected and minced in ice-cold PBS. After centrifugation $\left(4^{\circ} \mathrm{C}, 200 \times g, 5^{\prime}\right)$, tissues were cross-linked with $1 \%$ formaldehyde (Thermo Fisher Scientific, Cat\# 28906), 30' at RT. A total of 2.5 M glycine (MilliporeSigma, Cat\# G8898) was added at a final concentration $0.125 \mathrm{M}$ to stop the reaction. After the tissues were washed with $\mathrm{PBS}$, and centrifuged $\left(4^{\circ} \mathrm{C}, 200 \times g, 5^{\prime}\right)$, the pellets were frozen in dry ice and stored at $-80^{\circ} \mathrm{C}$. We used 20 embryos for one sample and performed three biological replicates. The frozen tissues were homogenized by BeadBug Microtube Homogenizer (Benchmark Scientific, Edison, NJ) in Lysis buffer, which contained $50 \mathrm{mM}$ Hepes pH 7.5 (MilliporeSigma, Cat\# H0887), $140 \mathrm{mM} \mathrm{NaCl}$ $1 \mathrm{mM}$ EDTA (MilliporeSigma, Cat\# E7889), 10\% Glycerol (MilliporeSigma, Cat\# 4750OP), 0.5\% IGEPAL CA-630 (MilliporeSigma, Cat\# I8896), 0.25\% TritonX-100 (MilliporeSigma, Cat\# X100) and Protease Inhibitor cocktail (MilliporeSigma, Cat\# P8340). Samples were incubated on ice for $10^{\prime}$. After centrifugation $\left(4^{\circ} \mathrm{C}, 2000 \times g, 5^{\prime}\right)$, extracted nuclei were washed in Wash buffer containing $10 \mathrm{mM}$ Tris- $\mathrm{HCl} \mathrm{pH} 8.0$ (MilliporeSigma, Cat\# T2694), $200 \mathrm{mM} \mathrm{NaCl}, 1 \mathrm{mM}$ EDTA pH 8.0, 0.5 mM EGTA pH 8.0 (MilliporeSigma, Cat\# E3889) and Protease Inhibitor Cocktail. Then the nuclei were resuspended in Shearing buffer containing $10 \mathrm{mM}$ Tris-HCl pH 8.0, $1 \mathrm{mM}$ EDTA pH 8.0, 0.1\% SDS (BIO-RAD, Cat\# 1610418), and Protease Inhibitor Cocktail. Resuspended nuclei were sonicated with a S2 Focused-ultrasonicator (Covaris, Inc., Woburn, MA). After sonication, $10 \%$ TritonX-100 and $5 \mathrm{M} \mathrm{NaCl}$ were added to sheared chromatin in a final concentration of $1 \%$ TritonX-100 and $150 \mathrm{mM} \mathrm{NaCl}$. Dynabeads ${ }^{\mathrm{st}} \mathrm{MyOne}^{\mathrm{m}} \mathrm{m}^{\mathrm{m}}$ Streptavidin T1 (Thermo Fisher Scientific, Cat\# 65602) were blocked with SEA BLOCK Blocking Buffer (Thermo Fisher Scientific, Cat\# 37527), and sheared chromatin was blocked with Dynabeads $s^{\text {mit }}$ Protein A for immunoprecipitation (Thermo Fisher Scientific, Cat\# 10002D) and Dynabeads ${ }^{\mathrm{m}}$ Protein G for immunoprecipitation (Thermo Fisher Scientific, Cat\# 10004D) at $4^{\circ} \mathrm{C}, 1 \mathrm{~h}$ on a rotator. Following washing with Shearing buffer, blocked streptavidin magnetic beads were added to the sheared chromatin, then incubated $4{ }^{\circ} \mathrm{C}$ overnight on a rotator. Streptavidin magnetic beads were washed with low-salt wash buffer $(20 \mathrm{mM}$ Tris- $\mathrm{HCl} \mathrm{pH} 8.0,45 \mathrm{mM} \mathrm{NaCl}, 0.1 \%$ SDS, $1 \%$ TritonX-100, $2 \mathrm{mM}$ EDTA), high-salt wash buffer (20 mM Tris-HCl pH 8.0, $150 \mathrm{mM} \mathrm{NaCl}, 0.1 \%$ SDS, $1 \%$ TritonX-100, $2 \mathrm{mM}$ EDTA), LiCl wash buffer containing $10 \mathrm{mM}$ Tris-HCl pH 8.0, $0.25 \mathrm{M} \mathrm{LiCl}$ (MilliporeSigma, Cat\# L7026), 1\% IGEPAL ${ }^{\circledR}$ CA$630,1 \%$ sodium deoxycholate (MilliporeSigma, Cat\# D6750), 1 mM EDTA. After a wash with TE buffer, DNA was eluted in Elution buffer $(10 \mathrm{mM}$ Tris- $\mathrm{HCl} \mathrm{pH} \mathrm{8.0,}$ $1 \mathrm{mM}$ EDTA, $1 \%$ SDS) with Proteinase K (Promega, Cat\# V3021) at $65^{\circ} \mathrm{C}$, $5 \mathrm{~h}$. Eluted DNA was purified with the MinElute PCR purification kit. After measuring the DNA concentration with the Qubit ${ }^{\oplus}$.0 Fluorometer using Qubit dsDNA HS Assay Kit, the libraries were prepared using Accel-NGS 2S Plus DNA Library Kit (Swift Bioscience, Cat\# 21024) and 2S Set A Indexing Kit (Swift Bioscience, Cat\# 26148); then the DNA concentration was measured with the Qubit ${ }^{\oplus} 2.0$ Fluorometer using Qubit dsDNA HS assay kit.

Sequencing. The DNA libraries were sequenced using an Illumina HiSeq2500 system (at Einstein Epigenomics Core Facility), Illumina HiSeq4000 system (at Genewiz, South Plainfield, NJ) or NovaSeq6000 system (at Novogene, Sacramento, CA), with paired-end, 100 bp read length.

RNAscope. RNAscope on tissue sections: After incubation of 4\% PFA (Alfa Aesar Cat\# 43368 ) at $4{ }^{\circ} \mathrm{C}$ for $4 \mathrm{~h}$, embryos were dehydrated with $70 \%, 90 \%$, and $100 \%$ Ethanol (Thermo Fisher Scientific, Cat\# A405P-4). Incubation with xylene (Thermo Fisher Scientific, Cat\# X3S-4) was done at RT, an hour twice followed by incubation of paraffin (Thermo Fisher Scientific, Cat\# T555) at $65^{\circ} \mathrm{C}$, an hour twice. Embryos were embedded and stored at $4{ }^{\circ} \mathrm{C}$.

Embryos were sectioned at 7- $\mu \mathrm{m}$ thickness and dried at RT. The sections were processed with RNAscope Multiplex Fluorescent v2 reagents (Advanced Cell Diagnostics, Cat\# 323100), according to the manufacturer's instructions. Briefly, sections were deparaffinized in xylene and dehydrated in graded ethanol, then incubated with hydrogen peroxide at RT, in $10^{\prime}$. The sections were incubated in boiled $1 \times$ Target retrieval reagent, for 15'. After rinsing with distilled water and $100 \%$ ethanol, the sections were dried at RT, for 5'. The sections were incubated with Protease Plus at RT, for 3' then incubated with mixed RNAscope probes of $\mathrm{C} 1, \mathrm{C} 2$, and $\mathrm{C} 3$ channels (Supplementary Table 2), at $40^{\circ} \mathrm{C}$, overnight. Following AMP1, AMP2, and AMP3 treatment, the sections were incubated with Opal 570 (AKOYA BIOSCIENCES, Cat\# FP1488001KT) for C1, Opal 620 (AKOYA BIOSCIENCES, Cat\# FP1495001KT) for C2, and Opal 520 (AKOYA BIOSCIENCES, Cat\# FP1487001KT) for C3 in 1:1000 dilution at RT, for 15'. Then slides were mounted with VECTASHIELD ${ }^{\circledast}$ HardSet $^{\mathrm{rt}}$ Antifade Mounting Medium with DAPI (Maravai LifeSciences, Cat\# H1500). Images were then captured using a Zeiss Axio Observer microscope with an ApoTome (Carl Zeiss Corp.).

RNAscope on whole embryos: Embryos were fixed in $4 \%$ PFA at $4{ }^{\circ} \mathrm{C}$ overnight, and dehydrated in $25 \%, 50 \%, 75 \%$, and $100 \%$ Methanol (Thermo Fisher scientific, A412-4). Embryos were stored at $-20^{\circ} \mathrm{C}$ until needed. Embryos were rehydrated in 
75\%, 50\%, 25\% methanol, and PBS $+0.1 \%$ Tween (Sigma, Cat\# P7949). The embryos were permeabilized using Protease III (Advanced Cell Diagnostics, Cat\# 323100 ) for $20^{\prime}$ followed by a wash with PBS $+0.01 \%$ Tween. The C1, C2, and C3 probes chosen for the experiments were warmed at $40^{\circ} \mathrm{C}$ for $10 \mathrm{~min}$. Embryos were incubated with $100 \mu \mathrm{L}$ of mixed RNAscope probes of C1, C2, and C3 (50:1:1, respectively) channels (Supplementary Table 2), at $40^{\circ} \mathrm{C}$, overnight. Three washes using $0.2 \times \mathrm{SSC}+0.01 \%$ Tween at RT were used in between all future steps. After washing, embryos were fixed for $10^{\prime}$ in $4 \%$ PFA at RT. Embryos were incubated in AMP1 for $30^{\prime}$, AMP2 for $30 \mathrm{~min}$, and AMP3 for $15 \mathrm{~min}$ at $40^{\circ} \mathrm{C}$ with washes in between. Tyramide Signal Amplification (TSA) was prepared at 1:2000 for TSA-CY3 (Akoya Biosciences, Cat\# NEL744001KT), 1:1000 for TSA-CY5 (Akoya Biosciences, Cat\# NEL745001KT), and 1:500 for TSA-Fluorescein (Akoya Biosciences, Cat\# NEL741001KT). Embryos were incubated in HRP-C1 for $15^{\prime}$ at $40^{\circ} \mathrm{C}$ followed by $30^{\prime}$ in their chosen TSA for C1. Amplification was blocked in HRP-Blocker for 15'. The previous two steps were then repeated for HRP-C2 for C2 probes and HRP-C3 for $\mathrm{C} 3$ probes. After the final washes, embryos were incubated in DAPI (Advanced Cell Diagnostics, Cat\# 323100) for 2 days then washed and stored at $4{ }^{\circ} \mathrm{C}$ until ready for imaging. Imaging was conducted with a confocal Leica SP5.

scRNA-seq data analysis. We utilized Cell Ranger (v 3.1.0, from 10x Genomics) to align reads of scRNA-seq data to the mouse reference genome (assembly, $\mathrm{mm} 10$ ). All the samples passed quality control measures for Cell Ranger (Table 1), and the filtered gene-barcode matrices were used for the following analyses. For Mesp1 $1^{C r e}$ four time point dataset analyses, Scran v1.10.2 was used to normalize the individual datasets by using the computeSumFactors function with the deconvolution method for scaling normalization ${ }^{79}$. The cells were clustered by densityClust v0.3 with the density peak clustering algorithm ${ }^{80}$. We found batch effects existed in the scRNA-seq datasets from different time points (Mesp1 $1^{\text {Cre }}$ data) or experimental perturbations ( $\mathrm{Mesp}_{1}{ }^{\mathrm{Cre}}$ or $\mathrm{Tb} \times 1^{\mathrm{Cre}} \mathrm{Ctrl}$ versus $\mathrm{cKO}$ ). Therefore, we performed batch corrections before we comprehensively analyzed gene expression values across these scRNA-seq datasets. We employed the MNN (mutual nearest neighbors) method to identify shared cell types across datasets, and corrected batches, according to the shared cell types, by the MNN batch correction method ${ }^{81}$. More specifically, we removed batch effects by fastMNN function of the Scran package, with the input of the normalized counts from individual datasets. The Mesp1 $1^{\text {Cre }}$ Ctrl data from different stages are comprised of homogeneous cell types (transcriptomes of each cell type are concordant across datasets), but the Mesp $1^{\text {Cre }} \mathrm{Ctrl}$ with cKO and then separately, the $\mathrm{Tb}_{x 1} \mathrm{Cre}$ Ctrl versus $\mathrm{CKO}$, are comprised of heterogeneous cell types (the same cell types from different conditions, Ctrl vs cKO, with dissimilar gene expression). Thus, we aligned gene expression values for Ctrl vs cKO data using the RPCI (reference principal component integration) method in RISC, which utilizes the global gene reference to calibrate the gene expression changes of heterogeneous cell types ${ }^{37}$. In detail, we combined individual datasets by the scMultiIntegrate function of the RISC package and outputted the corrected gene expression values after the clustering by Seurat 3.1.5 ${ }^{82}$. Then, the corrected datasets were processed on Scanpy v1.4.3 ${ }^{83}$ for cell trajectory analysis, with the PAGA approach ${ }^{26}$. The RISC software ${ }^{37}$ was also used to identify differentially expressed genes of Tbxl Ctrl vs cKO embryos in each cluster by a Negative Binomial generalized linear model ${ }^{84}$ (scDEG function of RISC package), with adjustive $P$ values $<0.05$ and $\operatorname{logFC}$ (log fold change) $>0.25$ or $<-0.25$. The clusterProfiler v3.10.1 ${ }^{85}$ was used for Gene Ontology pathway analysis, with adjustive $P$ values $<0.05$.

ATAC-seq data analysis. ATAC-seq analysis pipeline has been described previously ${ }^{86}$; however, we describe the methods here as well. We removed Nextera Transposase Sequence primers ${ }^{87}$ in the range $33-47$ bp using cutadapt ${ }^{88}$ with the following option -a CTGTCTCTTATACACATCTCCGAGCCCACGAGAC -A CTGTCTCTTATACACATCTGACGCTGCCGACGA. Sequences were then aligned to the mouse genome (mm9) using Bowtie2 2.3.4. $3^{89}$ with default parameters. Only uniquely mappable reads were retained. We removed reads with mates mapping to different chromosomes, or with discordant pairs orientation, or with a mate-pair distance $>2 \mathrm{~kb}$, or PCR duplicates (defined as when both mates are aligned to the same genomic coordinates). Reads mapping to the mitochondrial genome were also removed. ATAC peaks in each sample were identified using MACS2 2.1.2.190 with the option -- nomodel --shif100 --extsize 200. The differentially enriched regions (DARs) of $\mathrm{Tb}{ }_{1} \mathrm{Cre}$ Ctrl vs $\mathrm{cKO}$ were obtained using DiffBind $2.14 .0^{91}$ by loading all the MACS2 peaks, default parameters were used except for dba.count method where summits have been set to 250 . After that, we formed a consensus list of enriched regions for each condition using the intersectBed function from the BEDTools $2.29^{92}$ with the default minimum overlap and retaining only the peak regions common to all three replicates. We defined common peaks the regions that were common to the consensus peaks of both $\mathrm{Tbx} 1^{\mathrm{Cre}}$ $\mathrm{Ctrl}$ and $\mathrm{cKO}$ (using again the intersectBed function). The common peaks inTbxi ${ }^{C r e} \mathrm{Ctrl}$ vs cKO data that did not intersect the DARs were defined as common accessible regions (CARs). Both DARs and CARs were filtered out by removing peaks intersecting blacklist regions (Encode mm9 blackregions Version 2) using findOverlapsOfPeaks of ChIPpeakAnno v3.22.4 $4^{93}$ before any further analysis. Then DARs or CARs were filtered with the consensus Mesp1 Cre ATACseq peaks using intersectBed. Transcription factor binding motifs were obtained using the findMotifsGenome program of the HOMER suite (v4.10.3) $)^{94}$. For peak annotation as cis-regulatory regions, GREAT ${ }^{95}$ was used with default settings with $\mathrm{mm} 9$. The comparison of the gene list from DARs and DEGs was performed using standard R-scripts. The clusterProfiler v3.10.1 was used for Gene Ontology pathway analysis. Coverage heatmaps and average enrichment profiles (TSS + I$10 \mathrm{~Kb})$ in each experimental condition were obtained using ngs.plot ${ }^{96}$ or deepTools $2^{97}$. We applied statistical analysis in the processes of all the software and packages with default settings.

ChIP-seq data analysis. We removed adapters using cutadapt ${ }^{88}$ with -a option and a set of adapters detected with FASTQC ${ }^{98}$. Sequences were then trimmed using TrimGalore with option -length 0 . Then sequences were aligned to the mouse genome (mm9) using Bowtie2 2.3.4. with default parameters. Reads were removed with mates mapping to different chromosomes, or with discordant pairs orientation, or with a mate-pair distance $>2 \mathrm{~kb}$, or PCR duplicates (defined as when both mates are aligned to the same genomic coordinate). Only uniquely mappable reads were retained. ChIP-seq peaks in each sample were identified using MACS2 2.1.2.1 with default parameters. Then, a consensus list of enriched regions was obtained using the intersectBed function from the BEDTools 2.29 with the default minimum overlap and retaining only the peak regions common to at least two out of the three replicates. Peaks were filtered by removing those overlapping with blacklist regions (Encode mm9 black regions Version 2) using findOverlapsOfPeaks of ChIPpeakAnno. The transcription factor binding motifs were obtained using the findMotifsGenome program with -size given parameter of the HOMER suite. For peak annotation as cis-regulatory regions, GREAT was used with the default settings $\mathrm{mm}$. The comparison of the gene lists of DAR, DEG, and ChIP regions was performed using the dplyr package in R. IGV $2.4 .8^{97}$ was used for peak visualization. Coverage heatmaps and average enrichment profiles (TSS $+/-10 \mathrm{~Kb})$ in each experimental condition were obtained using ngs.plot or deepTools2. The significance of the overlap between the two lists of peaks was evaluated using the ChIPseeker enrichPeakOverlap using $\mathrm{mm} 9$ annotation. We applied statistical analysis in the processes of all the software and packages with default settings.

Quantification and statistical analysis. Besides the methods for scRNA-seq analysis, specific statistical tests were described in "Results" and Figure legends. Briefly, to determine the relative proportions of the CPM populations, a two proportion $Z$ test was performed in each cluster with $95 \%$ confidence interval. For ATAC-seq and TBX1 ChIP-seq, default statistical analysis was used for DiffBind, HOMER, and GREAT. The heatmap of ATAC-seq read densities in TBX1 ChIPseq peaks on ATAC-seq signals were determined using enrichPeakOverlap present in ChIPseeker. For Venn diagrams, Pearson's Chi-squared test was performed.

Reporting summary. Further information on research design is available in the Nature Research Reporting Summary linked to this article.

\section{Data availability}

The datasets generated during this study are available at the GEO repository under the accession numbers: scRNA-seq "GSE167493", scRNA-seq "GSE167491", ATAC-seq "GSE173700", and ChIP-seq "GSE173521". The scRNA-seq data can be viewed at https:// scviewer.shinyapps.io/heartMLP. All other relevant data supporting the key findings of this study are available within the article and its Supplementary Information files or from the corresponding author upon reasonable request.

Received: 8 January 2021; Accepted: 18 October 2021; Published online: 17 November 2021

\section{References}

1. Cai, C. L. et al. Isll identifies a cardiac progenitor population that proliferates prior to differentiation and contributes a majority of cells to the heart. Dev. Cell 5, 877-889 (2003).

2. Diogo, R. et al. A new heart for a new head in vertebrate cardiopharyngeal evolution. Nature 520, 466-473 (2015).

3. Kelly, R. G., Brown, N. A. \& Buckingham, M. E. The arterial pole of the mouse heart forms from Fgf10-expressing cells in pharyngeal mesoderm. Dev. Cell $\mathbf{1}$, 435-440 (2001).

4. Mjaatvedt, C. H. et al. The outflow tract of the heart is recruited from a novel heart-forming field. Dev. Biol. 238, 97-109 (2001).

5. Stefanovic, S. et al. Hox-dependent coordination of mouse cardiac progenitor cell patterning and differentiation. eLife 9 , https://doi.org/10.7554/eLife.55124 (2020)

6. Tian, Y. et al. Characterization and in vivo pharmacological rescue of a Wnt2Gata6 pathway required for cardiac inflow tract development. Dev. Cell 18, 275-287 (2010)

7. Saga, Y. et al. MesP1 is expressed in the heart precursor cells and required for the formation of a single heart tube. Development 126, 3437-3447 (1999). 
8. Lescroart, F. et al. Defining the earliest step of cardiovascular lineage segregation by single-cell RNA-seq. Science 359, 1177-1181 (2018).

9. Lescroart, F. et al. Clonal analysis reveals common lineage relationships between head muscles and second heart field derivatives in the mouse embryo. Development 137, 3269-3279 (2010).

10. Lescroart, F. et al. Clonal analysis reveals a common origin between nonsomite-derived neck muscles and heart myocardium. Proc. Natl Acad. Sci. USA 112, 1446-1451 (2015).

11. Nathan, E. et al. The contribution of Islet1-expressing splanchnic mesoderm cells to distinct branchiomeric muscles reveals significant heterogeneity in head muscle development. Development 135, 647-657 (2008).

12. Stolfi, A. et al. Early chordate origins of the vertebrate second heart field. Science 329, 565-568 (2010).

13. McDonald-McGinn, D. M. et al. 22q11.2 deletion syndrome. Nat. Rev. Dis. Prim. 1, 15071 (2015).

14. Kollara, L. et al. Velopharyngeal structural and muscle variations in children with 22q11.2 deletion syndrome: an unsedated MRI study. Cleft Palate Craniofac J. 56, 1139-1148 (2019).

15. Yagi, H. et al. Role of TBX1 in human del22q11.2 syndrome. Lancet 362 , 1366-1373 (2003).

16. Jerome, L. A. \& Papaioannou, V. E. DiGeorge syndrome phenotype in mice mutant for the T-box gene, Tbx1. Nat. Genet. 27, 286-291 (2001).

17. Merscher, S. et al. TBX1 is responsible for cardiovascular defects in velocardio-facial/DiGeorge syndrome. Cell 104, 619-629 (2001).

18. Lindsay, E. A. et al. Tbx1 haploinsufficieny in the DiGeorge syndrome region causes aortic arch defects in mice. Nature 410, 97-101 (2001).

19. Kelly, R. G., Jerome-Majewska, L. A. \& Papaioannou, V. E. The del22q11.2 candidate gene Tbxl regulates branchiomeric myogenesis. Hum. Mol. Genet. 13, 2829-2840 (2004).

20. Liao, J. et al. Identification of downstream genetic pathways of Tbx1 in the second heart field. Dev. Biol. 316, 524-537 (2008).

21. Kong, P. et al. Tbxl is required autonomously for cell survival and fate in the pharyngeal core mesoderm to form the muscles of mastication. Hum. Mol. Genet. 23, 4215-4231 (2014).

22. Harel, I. et al. Pharyngeal mesoderm regulatory network controls cardiac and head muscle morphogenesis. Proc. Natl Acad. Sci. USA 109, 18839-18844 (2012).

23. Hoffmann, A. D. et al. Foxf genes integrate tbx 5 and hedgehog pathways in the second heart field for cardiac septation. PLoS Genet. 10, e1004604 (2014).

24. Steimle, J. D. et al. Evolutionarily conserved Tbx5-Wnt2/2b pathway orchestrates cardiopulmonary development. Proc. Natl Acad. Sci. USA 115, E10615-E10624 (2018).

25. Srivastava, D. Making or breaking the heart: from lineage determination to morphogenesis. Cell 126, 1037-1048 (2006).

26. Wolf, F. A. et al. PAGA: graph abstraction reconciles clustering with trajectory inference through a topology preserving map of single cells. Genome Biol. 20, 59 (2019).

27. D'Aniello, C. et al. The G-protein-coupled receptor APJ is expressed in the second heart field and regulates Cerberus-Baf60c axis in embryonic stem cell cardiomyogenesis. Cardiovasc Res. 100, 95-104 (2013).

28. Lai, D. et al. Neuregulin 1 sustains the gene regulatory network in both trabecular and nontrabecular myocardium. Circ. Res. 107, 715-727 (2010).

29. Cortes, C., Francou, A., De Bono, C. \& Kelly, R. G. Epithelial properties of the second heart field. Circ. Res. 122, 142-154 (2018).

30. Meilhac, S. M. \& Buckingham, M. E. The deployment of cell lineages that form the mammalian heart. Nat. Rev. Cardiol. 15, 705-724 (2018).

31. Zhang, Z. et al. Tbxl expression in pharyngeal epithelia is necessary for pharyngeal arch artery. Dev. Dev. 132, 5307-5315 (2005).

32. Zhang, Z., Huynh, T. \& Baldini, A. Mesodermal expression of Tbx1 is necessary and sufficient for pharyngeal arch and cardiac outflow tract. Dev. Dev. 133, 3587-3595 (2006).

33. Chen, L., Fulcoli, F. G., Tang, S. \& Baldini, A. Tbx1 regulates proliferation and differentiation of multipotent heart progenitors. Circ. Res. 105, 842-851 (2009).

34. De Bono, C. et al. T-box genes and retinoic acid signaling regulate the segregation of arterial and venous pole progenitor cells in the murine second heart field. Hum. Mol. Genet. 27, 3747-3760 (2018).

35. Rana, M. S. et al. Tbxl coordinates addition of posterior second heart field progenitor cells to the arterial and venous poles of the heart. Circ. Res. 115, 790-799 (2014).

36. Hasten, E. \& Morrow, B. E. Tbx1 and Foxi3 genetically interact in the pharyngeal pouch endoderm in a mouse model for 22q11.2 deletion syndrome. PLoS Genet. 15, e1008301 (2019).

37. Liu, Y., Wang, T., Zhou, B. \& Zheng, D. Robust integration of multiple singlecell RNA sequencing datasets using a single reference space. Nat. Biotechnol. 39, 877-884 (2021).

38. Chen, Y. H., Ishii, M., Sun, J., Sucov, H. M. \& Maxson, R. E. Jr. Msx1 and Msx2 regulate survival of secondary heart field precursors and post-migratory proliferation of cardiac neural crest in the outflow tract. Dev. Biol. 308, 421-437 (2007).

39. Dodou, E., Verzi, M. P., Anderson, J. P., Xu, S. M. \& Black, B. L. Mef2c is a direct transcriptional target of ISL1 and GATA factors in the anterior heart field during mouse embryonic. Dev. Dev. 131, 3931-3942 (2004).

40. Fischer, A. et al. Combined loss of Heyl and HeyL causes congenital heart defects because of impaired epithelial to mesenchymal transition. Circ. Res. 100, 856-863 (2007).

41. Bai, Y. et al. Bmp signaling represses Vegfa to promote outflow tract cushion. Dev. Dev. 140, 3395-3402 (2013).

42. Cohen, E. D., Miller, M. F., Wang, Z., Moon, R. T. \& Morrisey, E. E. Wnt5a and Wnt11 are essential for second heart field progenitor development. Development 139, 1931-1940 (2012).

43. Sinha, T. et al. Loss of Wnt5a disrupts second heart field cell deployment and may contribute to OFT malformations in DiGeorge syndrome. Hum. Mol. Genet. 24, 1704-1716 (2015).

44. Scheper, V., Schwieger, J., Hamm, A., Lenarz, T. \& Hoffmann, A. BDNFoverexpressing human mesenchymal stem cells mediate increased neuronal protection in vitro. J. Neurosci. Res. 97, 1414-1429 (2019).

45. Wong, F. K. et al. Sustained Pax6 expression generates primate-like basal radial glia in developing mouse neocortex. PLoS Biol. 13, e1002217 (2015).

46. Plachov, D. et al. Pax8, a murine paired box gene expressed in the developing excretory system and thyroid gland. Development 110, 643-651 (1990).

47. Vitelli, F. et al. A genetic link between Tbx1 and fibroblast growth factor signaling. Development 129, 4605-4611 (2002).

48. Guo, C. et al. A Tbx1-Six1/Eya1-Fgf8 genetic pathway controls mammalian cardiovascular and craniofacial morphogenesis. J. Clin. Investig. 121, 1585-1595 (2011)

49. Gorkin, D. U. et al. Author Correction: An atlas of dynamic chromatin landscapes in mouse fetal development. Nature 589, E4 (2021).

50. Francou, A. et al. Second heart field cardiac progenitor cells in the early mouse embryo. Biochim Biophys. Acta 1833, 795-798 (2013).

51. Soh, B. S. et al. N-cadherin prevents the premature differentiation of anterior heart field progenitors in the pharyngeal mesodermal microenvironment. Cell Res. 24, 1420-1432 (2014).

52. Sinha, T., Wang, B., Evans, S., Wynshaw-Boris, A. \& Wang, J. Disheveled mediated planar cell polarity signaling is required in the second heart field lineage for outflow tract morphogenesis. Dev. Biol. 370, 135-144 (2012).

53. Francou, A., Saint-Michel, E., Mesbah, K. \& Kelly, R. G. TBX1 regulates epithelial polarity and dynamic basal filopodia in the second heart field. Development 141, 4320-4331 (2014).

54. Racedo, S. E. et al. Reduced dosage of beta-catenin provides significant rescue of cardiac outflow tract anomalies in a Tbx1 conditional null mouse model of 22q11.2 deletion syndrome. PLoS Genet. 13, e1006687 (2017).

55. Moncaut, N. et al. Musculin and TCF21 coordinate the maintenance of myogenic regulatory factor expression levels during mouse craniofacial development. Development 139, 958-967 (2012).

56. Dong, F. et al. Pitx2 promotes development of splanchnic mesoderm-derived branchiomeric muscle. Development 133, 4891-4899 (2006).

57. Razy-Krajka, F. et al. Collier/OLF/EBF-dependent transcriptional dynamics control pharyngeal muscle specification from primed cardiopharyngeal progenitors. Dev. Cell 29, 263-276 (2014).

58. Adachi, N., Bilio, M., Baldini, A. \& Kelly, R. G. Cardiopharyngeal mesoderm origins of musculoskeletal and connective tissues in the mammalian pharynx. Development 147, https://doi.org/10.1242/dev.185256 (2020).

59. Taddei, I., Morishima, M., Huynh, T. \& Lindsay, E. A. Genetic factors are major determinants of phenotypic variability in a mouse model of the DiGeorge/ del22q11 syndromes. Proc. Natl Acad. Sci. USA 98, 11428-11431 (2001).

60. Kuba, K., Sato, T., Imai, Y. \& Yamaguchi, T. Apelin and Elabela/Toddler; double ligands for APJ/Apelin receptor in heart development, physiology, and pathology. Peptides 111, 62-70 (2019).

61. Scott, I. C. et al. The g protein-coupled receptor agtrllb regulates early development of myocardial progenitors. Dev. Cell 12, 403-413 (2007).

62. Zeng, X. X., Wilm, T. P., Sepich, D. S. \& Solnica-Krezel, L. Apelin and its receptor control heart field formation during zebrafish gastrulation. Dev. Cell 12, 391-402 (2007).

63. Freyer, L. et al. Loss of apela peptide in mice causes low penetrance embryonic lethality and defects in early mesodermal derivatives. Cell Rep. 20, 2116-2130 (2017).

64. Meyer, D. \& Birchmeier, C. Multiple essential functions of neuregulin in development. Nature 378, 386-390 (1995).

65. Comai, G. et al. A distinct cardiopharyngeal mesoderm genetic hierarchy establishes antero-posterior patterning of esophagus striated muscle. eLife $\mathbf{8}$, https://doi.org/10.7554/eLife.47460 (2019).

66. Seo, S. \& Kume, T. Forkhead transcription factors, Foxc1 and Foxc2, are required for the morphogenesis of the cardiac outflow tract. Dev. Biol. 296, 421-436 (2006). 
67. Yamagishi, H. et al. Tbx1 is regulated by tissue-specific forkhead proteins through a common Sonic hedgehog-responsive enhancer. Genes Dev. 17, 269-281 (2003).

68. Nowotschin, S. et al. Tbx1 affects asymmetric cardiac morphogenesis by regulating Pitx2 in the secondary heart field. Development 133, 1565-1573 (2006).

69. Chen, L. et al. Transcriptional control in cardiac progenitors: Tbxl interacts with the BAF chromatin remodeling complex and regulates Wnt5a. PLoS Genet. 8, e1002571 (2012).

70. Watanabe, Y. et al. Fibroblast growth factor 10 gene regulation in the second heart field by Tbx1, Nkx2-5, and Islet1 reveals a genetic switch for down-regulation in the myocardium. Proc. Natl Acad. Sci. USA 109, 18273-18280 (2012).

71. $\mathrm{Xu}, \mathrm{H}$. et al. Tbxl has a dual role in the morphogenesis of the cardiac outflow tract. Development 131, 3217-3227 (2004).

72. Kodo, K., Uchida, K. \& Yamagishi, H. Genetic and cellular interaction during cardiovascular development implicated in congenital heart diseases. Front. Cardiovasc Med. 8, 653244 (2021).

73. Calmont, A. et al. Tbxl controls cardiac neural crest cell migration during arch artery development by regulating Gbx2 expression in the pharyngeal ectoderm. Development 136, 3173-3183 (2009).

74. Kelly, R. G. \& Papaioannou, V. E. Visualization of outflow tract development in the absence of Tbx1 using an FgF10 enhancer trap transgene. Dev. Dyn. 236, 821-828 (2007).

75. Huynh, T., Chen, L., Terrell, P. \& Baldini, A. A fate map of Tbx1 expressing cells reveals heterogeneity in the second cardiac field. Genesis 45, 470-475 (2007).

76. Arnold, J. S. et al. Inactivation of Tbx1 in the pharyngeal endoderm results in 22q11DS malformations. Development 133, 977-987 (2006).

77. Sousa, V. H., Miyoshi, G., Hjerling-Leffler, J., Karayannis, T. \& Fishell, G. Characterization of Nkx6-2-derived neocortical interneuron lineages. Cereb. Cortex 19(Suppl 1), i1-i10 (2009).

78. Buenrostro, J. D., Giresi, P. G., Zaba, L. C., Chang, H. Y. \& Greenleaf, W. J. Transposition of native chromatin for fast and sensitive epigenomic profiling of open chromatin, DNA-binding proteins and nucleosome position. Nat. Methods 10, 1213-1218 (2013).

79. Lun, A. T., McCarthy, D. J. \& Marioni, J. C. A step-by-step workflow for low-level analysis of single-cell RNA-seq data with Bioconductor. F1000Res 5, 2122 (2016).

80. Rodriguez, A. \& Laio, A. Machine learning. Clustering by fast search and find of density peaks. Science 344, 1492-1496 (2014).

81. Haghverdi, L., Lun, A. T. L., Morgan, M. D. \& Marioni, J. C. Batch effects in single-cell RNA-sequencing data are corrected by matching mutual nearest neighbors. Nat. Biotechnol. 36, 421-427 (2018).

82. Butler, A., Hoffman, P., Smibert, P., Papalexi, E. \& Satija, R. Integrating singlecell transcriptomic data across different conditions, technologies, and species. Nat. Biotechnol. 36, 411-420 (2018).

83. Wolf, F. A., Angerer, P. \& Theis, F. J. SCANPY: large-scale single-cell gene expression data analysis. Genome Biol. 19, 15 (2018).

84. Venables, W. N. \& Ripley, B. D. in Modern Applied Statistics with S (eds Chambers, J., Eddy, W., Hardle, W., Sheather, S. \& Tierney L.) 271-300 (Springer New York, 2002).

85. Yu, G., Wang, L. G., Han, Y. \& He, Q. Y. clusterProfiler: an R package for comparing biological themes among gene clusters. OMICS 16, 284-287 (2012).

86. Cirino, A. et al. Chromatin and transcriptional response to loss of TBX1 in early differentiation of mouse cells. Front. Cell Dev. Biol. 8, 571501 (2020).

87. Turner, F. S. Assessment of insert sizes and adapter content in fastq data from NexteraXT libraries. Front. Genet. 5, 5 (2014).

88. Martin, M. Cutadapt removes adapter sequences from high-throughput sequencing reads. eMBNetJ 17, 3 https://doi.org/10.14806/ej.17.1.200 (2011).

89. Langmead, B. \& Salzberg, S. L. Fast gapped-read alignment with Bowtie 2. Nat. Methods 9, 357-359 (2012).

90. Feng, J., Liu, T., Qin, B., Zhang, Y. \& Liu, X. S. Identifying ChIP-seq enrichment using MACS. Nat. Protoc. 7, 1728-1740 (2012)

91. Ross-Innes, C. S. et al. Differential oestrogen receptor binding is associated with clinical outcome in breast cancer. Nature 481, 389-393 (2012).

92. Quinlan, A. R. \& Hall, I. M. BEDTools: a flexible suite of utilities for comparing genomic features. Bioinformatics 26, 841-842 (2010).

93. Zhu, L. J. et al. ChIPpeakAnno: a Bioconductor package to annotate ChIP-seq and ChIP-chip data. BMC Bioinforma. 11, 237 (2010).

94. Heinz, S. et al. Simple combinations of lineage-determining transcription factors prime cis-regulatory elements required for macrophage and B cell identities. Mol. Cell 38, 576-589 (2010).

95. McLean, C. Y. et al. GREAT improves functional interpretation of cisregulatory regions. Nat. Biotechnol. 28, 495-501 (2010).
96. Shen, L., Shao, N., Liu, X. \& Nestler, E. ngs.plot: Quick mining and visualization of next-generation sequencing data by integrating genomic databases. BMC Genomics 15, 284 (2014).

97. Ramirez, F. et al. deepTools2: a next generation web server for deepsequencing data analysis. Nucleic Acids Res. 44, W160-W165 (2016).

98. Andrews, S. FastQC: a quality control tool for high throughput sequence data. http://www.bioinformatics.babraham.ac.uk/projects/fastqc (2010).

\section{Acknowledgements}

We thank Genomics core, especially David Reynolds, Director of the Genomics Core, Shahina Maqbool, Director of the Epigenomics core as well as the Flow Cytometry and Gene modification facilities at Einstein. We appreciate all the help in data analysis and training by Masako Suzuki at Einstein. We thank Drs. Bin Zhou, Cedric Blanpain, and Peter Scambler for reading this manuscript and providing helpful suggestions. We also thank NYU Center for Genomics and Systems Biology Genomics Core; NYU Langone's Genome Technology Center is partially supported by the Cancer Center Support Grant P30CA016087 at the Laura and Isaac Perlmutter Cancer Center, especially Peter Meyn. This work was supported by grants from the National Institutes of Health P01HD070454 (B.E.M. and D.Z.), R01HL153920 (B.E.M. and D.Z.), R01HD096770 (L.C.), and R01HL108643 (L.C.). The work was also supported by a grant from the Foundation Leducq (Transatlantic Network of Excellence 15CVD01; B.E.M., L.C., R.G.K., and A.B.) and the Agence Nationale de la Recherche Heartbox and Myohead projects (RGK). Dr. Nomaru was supported by an American Association Grant (19POST34380281).

\section{Author contributions}

H.N., L.C., R.G.K., A.B., D.Z., and B.E.M. designed the studies. H.N., Y.L., C.D.B., D.R., A.C., W.W., H.S., S.E.R., A.G.D., C.A., R.G.K., A.B., D.Z., and B.E.M. performed and analyzed the experiments. H.N. and B.E.M. wrote the paper. Y.L. and D.Z. provided expert bioinformatics knowledge and guidance with the intellectual contribution of biological interpretation from C.D.B. and R.G.K. C.A. provided expert knowledge for ATAC-seq and ChIP-seq with the consultation of D.Z. L.Z. and C.-L.C. provided the conceptional knowledge and helped generate Tbx1-Avi mice used for ChIP-seq. H.N. with H.S. performed RNAscope experiments and analysis with intellectual contribution by C.D.B. All authors reviewed the results, helped edit the manuscript, and approved the paper.

\section{Competing interests}

The authors declare no competing interests.

\section{Additional information}

Supplementary information The online version contains supplementary material available at https://doi.org/10.1038/s41467-021-26966-6.

Correspondence and requests for materials should be addressed to Bernice E. Morrow.

Peer review information Nature Communications thanks Simon Bamforth and the other anonymous reviewer(s) for their contribution to the peer review this work. Peer reviewer reports are available.

Reprints and permission information is available at http://www.nature.com/reprints

Publisher's note Springer Nature remains neutral with regard to jurisdictional claims in published maps and institutional affiliations.

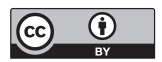

Open Access This article is licensed under a Creative Commons Attribution 4.0 International License, which permits use, sharing adaptation, distribution and reproduction in any medium or format, as long as you give appropriate credit to the original author(s) and the source, provide a link to the Creative Commons license, and indicate if changes were made. The images or other third party material in this article are included in the article's Creative Commons license, unless indicated otherwise in a credit line to the material. If material is not included in the article's Creative Commons license and your intended use is not permitted by statutory regulation or exceeds the permitted use, you will need to obtain permission directly from the copyright holder. To view a copy of this license, visit http://creativecommons.org/ licenses/by/4.0/.

(C) The Author(s) 2021 